Portland State University

PDXScholar

6-14-2019

\title{
Bleached Chondrules and the Possible Influence of Aqueous Alteration
}

Robert P. Kostynick

Portland State University, robert27@pdx.edu

Follow this and additional works at: https://pdxscholar.library.pdx.edu/geology_honorstheses

Part of the Geology Commons

Let us know how access to this document benefits you.

\section{Recommended Citation}

Kostynick, Robert P., "Bleached Chondrules and the Possible Influence of Aqueous Alteration" (2019). Geology Undergraduate Honors Theses. 1.

https://pdxscholar.library.pdx.edu/geology_honorstheses/1

This Thesis is brought to you for free and open access. It has been accepted for inclusion in Geology Undergraduate Honors Theses by an authorized administrator of PDXScholar. Please contact us if we can make this document more accessible: pdxscholar@pdx.edu. 
Honors Thesis

Bleached Chondrules and the Possible Influence of Aqueous Alteration

\author{
Submitted to \\ Department of Geology \\ Portland State University
}

By

Robert Kostynick

$06 / 14 / 2019$

Approval From:

Alexander Ruzicka, Thesis Advisor

Date

Martin Streck, Department Chair

Date 


\begin{abstract}
:
Aqueous alteration, chemical or petrologic change through the addition of water, is a proposed secondary effect of ordinary chondrites. Some minimally metamorphosed chondrites have been noted to have strong signs of aqueous alteration (Weisberg et al., 2006), but the degree of the effect in other chondrites is not clear. There is a specific chondrule type called "bleached chondrules" in which aqueous alteration is proposed to occur in a widespread fashion for all metamorphic grades (Grossman et al., 2000). Using transmitted optical microscopy (OM) and scanning electron microscopy (SEM) at Portland State University, this report obtained chemical and petrological data of nine select meteoritic bleached chondrules to test if the findings in Grossman et al. (2000) holds true for chondrites NWA 11991 (LL3.5) and Tieschitz (H/L3.6). Chondrules 4, 7, and 8, agreed with Grossman et al. (2000) in optical imagery, porosity, and bulk/subset chemical data. Chondrules 2, 3, and 5, agreed with optical imagery and bulk/subset chemical data but did not agree with the expected porosity. Chondrules 1, 6, and 9, did not agree with Grossman et al. (2000) and deviate from one another. Based on these findings, it is determined that aqueous alterations of the sort described by Grossman et al. does not fully describe bleaching process and that additional processes are necessary for bleaching. There is evidence within the findings that dissolution of the mesostasis occurred but due the inconsistency surrounding the solubility of elements, a physical removal of the mesostasis had to occur.
\end{abstract}

\title{
Introduction:
}

Meteorites, rocks that enter and survive earth's atmosphere, have been primary tools in understanding the formation of the solar system and early developments of geologic history. A large percentage of meteorites are considered to be chondritic, in which small spherical rocks called chondrules are present throughout (Scott et al., 1984). Chondrites are common sedimentary rocks with a fine-grained matrix surrounding spherical chondrules (Grossman et al., 2000). Most meteorites have been identified as originating from sub-planetary sized objects and a remaining small amount originated from planets or moons (Burbine et al. 2002). These subplanetary sized objects are generally asteroids which are thought to be the remains of planetesimals that helped shaped the current planetary system (Klarh and Schreiber, 2016). These asteroids in which a meteorite originates from are considered to be a parent body of the meteorites (Burbine et al. 2002). They are important components to the development of meteorites as they allow for a region of development and alteration to occur. The chondrules that make up chondrites formed in a solar nebula as melted clumps of dust (Hubbard \& Ebel, 2015) and eventually combined together in a parent body system (Scott \& Krot, 2003). Within these parent body systems, chondrites experienced various degrees of thermal metamorphism (Friedrich et al., 2014) and some may have even experienced aqueous alteration (Jilly-Rehak et al., 2017). Aqueous alteration is important in parent bodies and in chondrites as it can redistribute chemical information and produce a wide range of secondary phases through the influence of primitive water (Brearley, 2006). The higher the degree of metamorphism though, the less likely remaining evidence of aqueous alteration is seen and is thus rather limited to lowmetamorphosed chondrites (Jilly-Rehak et al., 2017, Grossman et al., 2000).

Bleached chondrules, a chondrule that may have experienced aqueous alteration and are the main focus of this report are normally seen in ordinary chondrites. Ordinary chondrites are the most common type of meteorite and are identified by having large chondrules as well as a $10-15 \%$ total matrix composition (Weisberg et al., 2006). These chondrites are commonly divided into 
greater branches of classification ranging from H, L, and LL. These classifications are generally used to describe a change in metal abundance and chondrule size (Weisberg et al., 2006). The metamorphic grade of chondrites is described with a petrographic scheme ranging from type 3 to type 6 chondrites (Grossman \& Brearley, 2005). Type 3 chondrites are meteorites that have experienced the least amount metamorphism and are often referred to as unequilibrated throughout most literature (Grossman \& Brearley, 2005). Two common chondrule textural types are radial pyroxene (RP) and cryptocrystalline (CC) chondrules (Grossman, 1998). RP chondrules are relatively non-porphyritic and have a single starting point of nucleation on the edges of the chondrules (Weyrauch \& Bischoff, 2013). These melt droplets or nucleation points cooled at a quick rate and in a fan like formation formed as crystallized pyroxene as seen in Figure 1 (Grossman, 1998). Normally, the space within the fan blades are filled with glassy mesostasis (Weyrauch \& Bischoff, 2013). CC chondrules are also non-porphyritic but crystals are so fine-grained that they cannot be readily distinguished with an optical microscope (Weyrauch \& Bischoff, 2013). Such chondrules also can contain glass (Grossman, 1998). These two types of chondrules are the most common chondrules to be subjected to bleaching (Grossman, 1998). Bleaching, a process in which physical and chemical alteration occurs in the rim of a chondrule, is assumed to be caused by the influence of aqueous alteration occurring in the chondrule (Grossman et al., 2000). These two types of chondrules, as well as any others that appear to be bleached are the primary tools used to understand how this alteration occurs. Bleached chondrules have been seen in meteorites of higher-grade metamorphism which is important to note when understanding the timeline of bleaching events (Grossman et al., 2000). It has been proposed that this means aqueous alteration had to occur before thermal metamorphism (Grossman et al., 2000).

To best understand if aqueous alteration does play an important role in the formation of these bleached chondrules, two chondritic meteorites were analyzed to see if Grossman et al.'s hypothesis would hold true for additional chondrites. The purpose of this report is to see how these bleached chondrules within the chondrites, resemble or derivate from Grossman et al.'s findings. The testing will give insight into whether these findings adequately characterize aqueous alteration.

\section{Background:}

The term bleached chondrules was first coined by Skinner et al. (1989). The description of a bleached chondrule at the time, was a chondrule that had experienced a leaching process (Skinner et al. 1989). This leaching process described is related to the loss of elements such as K, $\mathrm{Na}, \mathrm{Si}$ and $\mathrm{Al}$, most of which are concentrated in feldspathic glass (Grossman et al., 2000 \& Skinner et al. 1989).

There are many observations made by Grossman et al. (2000) of how this bleaching process occurred and its byproducts. The most important observations and interpretations made by Grossman et al. (2000) are listed below:

1. Bleached chondrules can be seen optically by having textural differences between core and rim. These boundaries between the core and rim can mimic the outer shape of chondrules.

2. Bleached chondrules are exclusively seen in cryptocrystalline and radial pyroxene chondrules. 
3. The rims are more porous than the cores in which likely indicates mesostasis has been lost.

4. Bleached chondrules are commonly seen in all metamorphic grades 3-6 but are seen in greater abundance in all type 3 chondrules.

5. Bleached chondrules are seen in all type 3 unequilibrated chondrules.

6. The abundance of these chondrules is greatest in $\mathrm{L}$ chondrules, then LL, and lowest in $\mathrm{H}$ chondrules.

7. Sulfide grains can be seen in some bleached rims as a byproduct of the bleaching process.

8. Calcium rich rims increase in quantity with increasing metamorphic grade.

9. Rims have a higher $\mathrm{H}_{2} \mathrm{O}$ content than core due to aqueous alteration.

10. Chondrule mesostases in the rim have been replaced by voids and/or hydrous phases.

11. Fe and $\mathrm{Mg}$ show little variations from rim to core in unequilibrated type 3 chondrules, but this variation tends to increase with increasing metamorphic grade.

12. $\mathrm{Na}, \mathrm{K}, \mathrm{Sr}, \mathrm{Si}$, and sometimes $\mathrm{Al}$, are removed from the rim while $\mathrm{H}_{2} \mathrm{O}, \mathrm{Fe}, \mathrm{Ni}$, Halogens, and $\mathrm{Rb}$ can be introduced as exchange elements through the bleaching process.

The aqueous alteration envisioned by Grossman et al. (2000) was thought to be sourced from melted ice in the parent body or by the oxidation of organic matter that would produce water within the system (Grossman et al., 2000). This aqueous alteration is inferred to occur during diagenesis, with dissolution of chondrule mesostasis glass resulting in an increase of porosity (Grossman et al., 2000 \& Skinner et al. 1989). Goudy and Koolhoven (2018), in preliminary work done at Portland State University, found deviations from some of these findings. Goudy and Koolhoven found that bleached zones of their samples tend to typically have lower porosity, not higher porosity. Further, chemical gradients and mesostasis textures sometimes differ from those described by Grossman et al. (2000).

This study examined nine bleached chondrules samples, five from chondrite NWA 11991 (LL3.5), sample 0091-3 [Figure 1] (the same sample that Goudy and Koolhoven studied), and four from Tieschitz (H/L3.6), sample 0781-A [Figure 2]. These meteorites were used to evaluate the findings of Grossman et al. (2000). The main emphasis of this report is to use a variant of the approach used by Grossman et al. (2000) to obtain bulk composition of the core and rims in which it could compared to element solubility. Whereas these authors used electron microprobe traverses to determine rim and core compositions, extracts of large rim and core areas from SEM $\mathrm{X}$-ray maps were used here. The bulk data was then compared to subset data analysis in which multiple small samples of similar size were compared in both the rim and the core. This was done to evaluate spatial variability of the Grossman approach. In addition, petrographic data appearance, relative porosity of rim and cores, and a comparison of $\mathrm{Fe} /(\mathrm{Fe}+\mathrm{Mg})$ content to $\mathrm{Al}$ as well as $\mathrm{Na}$ to $\mathrm{Al}$ to see how they vary in terms of solubility. 


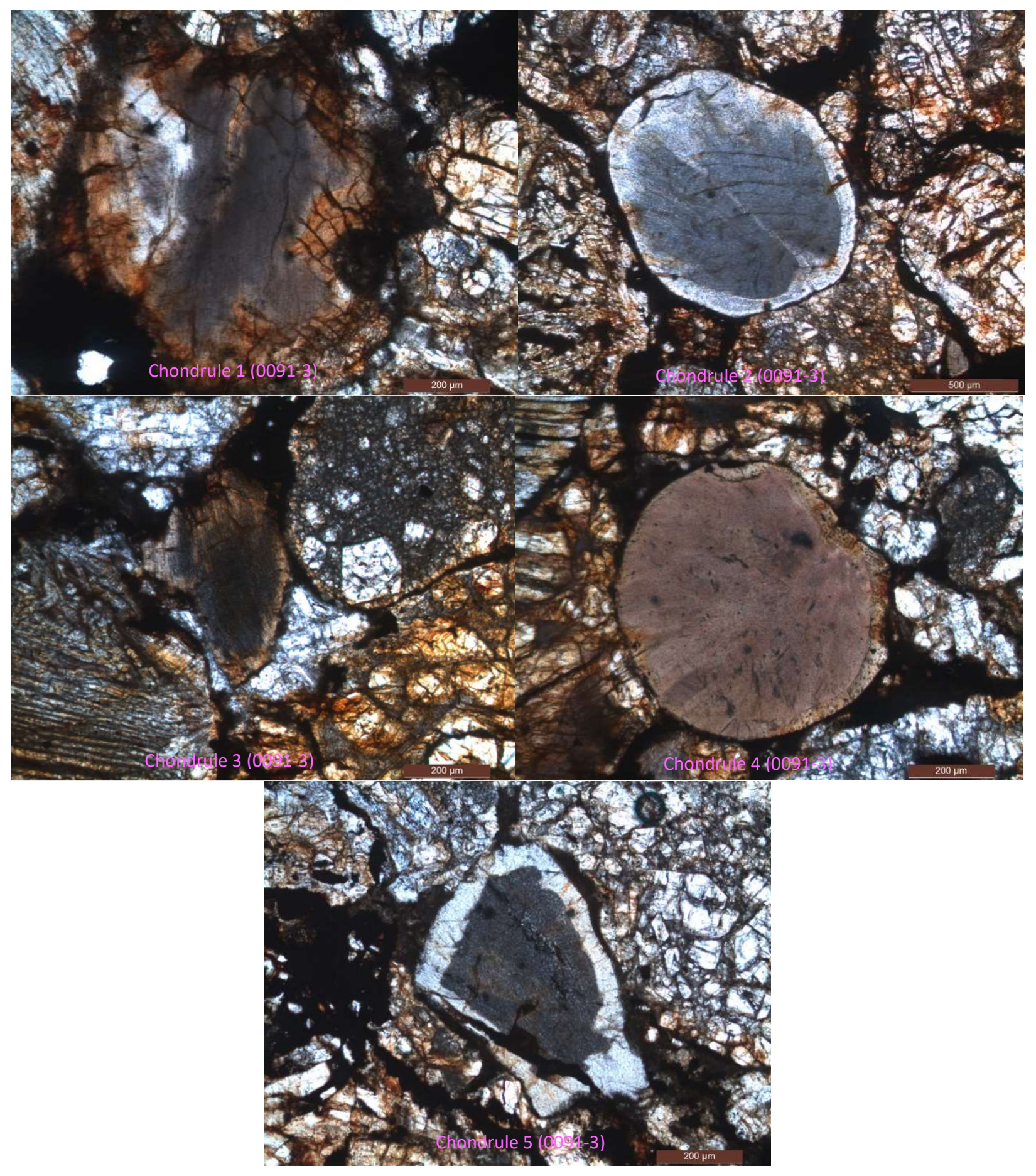

Figure 1: Optical Images of bleached chondrules found in chondrite sample 0091-3. Chondrules 1, 2, 3, and 5 are all radial pyroxene while chondrule 4 is a cryptocrystalline chondrule. Chondrule 2, 4, and 5 show ideal optical bleaching as the rims are clearly more transparent than the cores. 


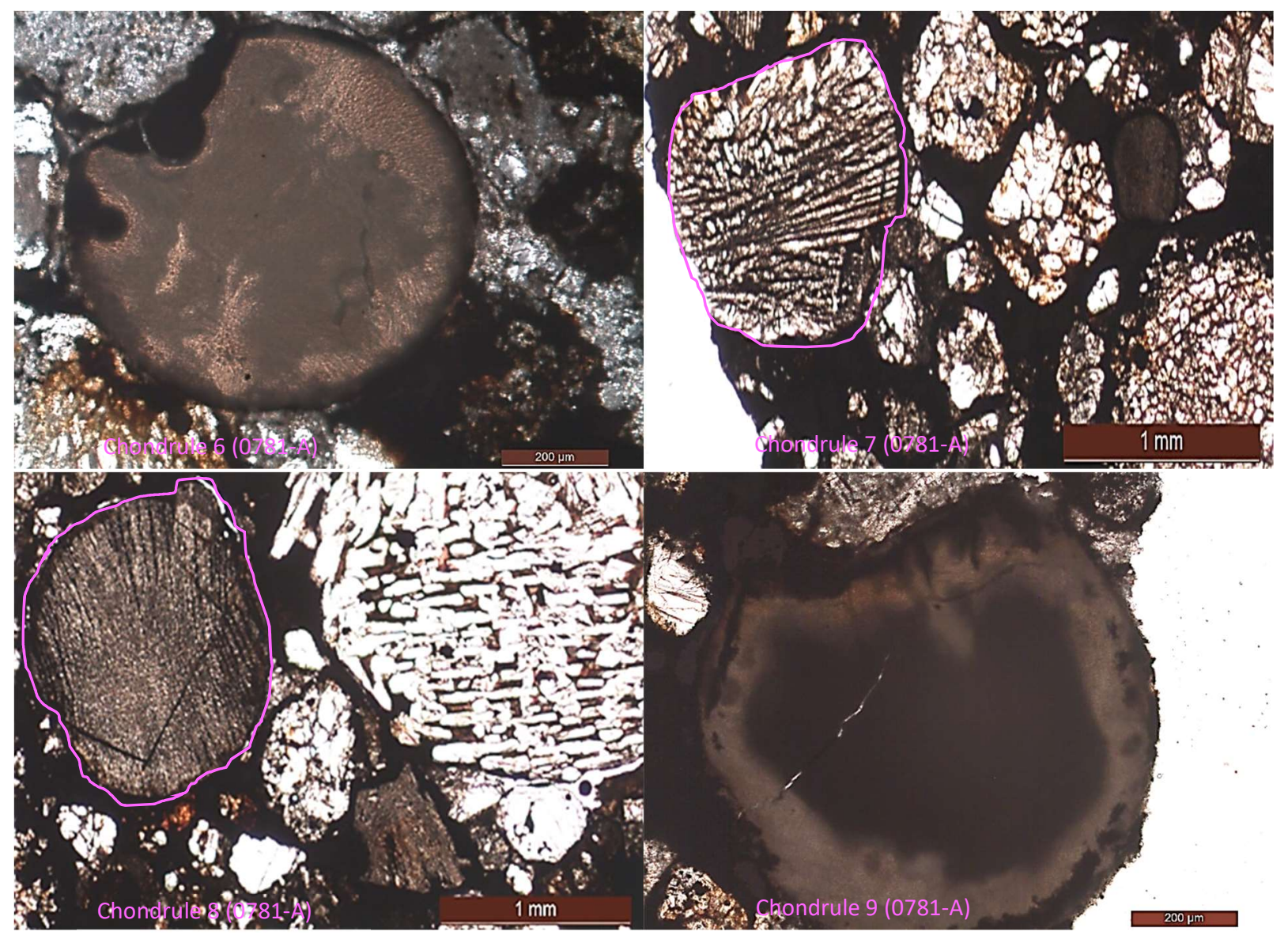

Figure 2: Optical Images of bleached chondrules found in chondrite sample 0781-A. Chondrules 7 and 8 are radial pyroxene while chondrule 6 and 9 are cryptocrystalline chondrules. Compared to chondrules 1-5, these chondrules do not exhibit as welldefined bleaching which was a common trend throughout sample 0781.

\section{Methods:}

\section{Optical Microscopy:}

To evaluate Grossman et al.'s hypothesis, bleached chondrules were located in the samples. Chondrules fitting the description of bleached chondrules as given by Grossman et al. (2000) were first located and imaged using a Leica DM2500 petrographic microscope. All samples had rather clear boundaries between the core and rim following in suit with Grossman et al. [Figure 1 \& Figure 2]. Some chondrules that were considered possible bleached chondrules based on optical appearance were removed from consideration following SEM imaging. Approximate sizes of the chondrules were found by using Adobe Photoshop and measuring two axes that were approximately $90^{\circ}$ degrees apart from each other. The measured lengths of the axes [Table 1] are shown in units of microns and are based on the calibrated scale bars seen in Figure 1 and Figure 2 . 
Table 1: The diameters of studied chondrules as measured in two axes roughly 90 degrees apart. These measurements were determined using the scale bar on the optical image and the ruler tool in Adobe Photoshop.

\begin{tabular}{|c|c|c|}
\hline $\begin{array}{c}\text { Chondrule and Section } \\
\text { Designation }\end{array}$ & Axis 1 $(\mu \mathrm{m})$ & Axis 2 $(\mu \mathrm{m})$ \\
\hline Chondrule 1 (0091-3) & 835 & 671 \\
\hline Chondrule 2 (0091-3) & 1246 & 1022 \\
\hline Chondrule 3 (0091-3) & 417 & 283.6 \\
\hline Chondrule 4 (0091-3) & 652.8 & 596.2 \\
\hline Chondrule 5 (0091-3) & 626.9 & 373.1 \\
\hline Chondrule 6 (0781-A) & 731 & 656.7 \\
\hline Chondrule 7 (0781-A) & 1164 & 821 \\
\hline Chondrule 8 (0781-A) & 1194 & 1044.7 \\
\hline Chondrule 9 (0781-A) & 783.6 & 708.9 \\
\hline
\end{tabular}

\section{Scanning Electron Microscopy:}

To prepare the thin sections for SEM analysis, a carbon layer of $\approx 10 \mathrm{~nm}$ was coated on each thin section. Samples were studied with a Zeiss Sigma FE-VP Scanning Electron Microscope at Portland State University using a $20 \mathrm{kV}$ accelerating voltage under high vacuum, using backscattered electron (BSE) images and energy dispersive spectrometer (EDS) methods including EDS mapping. Oxford Instruments AZtec software was used to obtain and process all SEM data. Map data were acquired in $\sim 20$ minute analyses of a raster areas, whereas point analyses were obtained with 25 second counts. Quantitative analyses were obtained by using factory calibrations and beam optimization on a $\mathrm{Cu}$ standard. Oxygen was calculated by stoichiometry assuming standard valences of cations, with valences of $\mathrm{Ni}$ and $\mathrm{S}$ set to zero for the purpose of calculating oxygen contents. Threshold (background) values for all analytical data were set at 1-Sigma uncertainty. EDS map data (1024 x 778 pixels) were combined with BSE images (2048 x 1556 pixels) to create false color maps by assigning different colors to different elements (Ni dark blue, Fe light blue, Cr fuschia, Ca red, $\mathrm{P}$ and $\mathrm{S}$ yellow, Si magenta, Al sky blue, Mg light green) [Figure 3]. Three SEM sessions were used to obtain all BSE and EDS data for the nine chondrules of this study.

\section{Bulk analysis:}

Bulk chemical compositions were obtained for bleached chondrule rims and cores using EDS map data by taking data extracts from regions that appeared chemically different in false color maps [Figure 3]. Using these elemental color maps, clear borders could be defined between rim and cores of the chondrule and allowed for weight, oxide, and atomic percentages to be obtained for both regions. Table 2 shows the compiled oxide $\%$ values of the bulk analysis.

The primary goal of the bulk sampling was to see how these bulk measurements varied with each element and how the data related to aqueous alteration. To devise a useful relationship of bulk measurement to aqueous alteration, the ionic potential (where ionic potential=charge of ion/ionic radius) was used for each element as a gauge of solubility. Low and high ionic potential represented elevated solubility (Buggle et al., 2011), which is consistent with observations for mobility of elements during terrestrial weathering (Li, 2000). Element mobility during terrestrial weathering depends on fluid composition, $\mathrm{pH}$, redox conditions, and temperature, but for near- 
neutral and oxidizing conditions element mobility increases in the approximate sequence: $\mathrm{Al} \sim$ $\mathrm{Ti} \sim \mathrm{Mn}<\sim \mathrm{Si}$ (intermediate ionic potential) $<\mathrm{P} \sim \mathrm{Cr} \sim \mathrm{S}$ (high ionic potential) $\sim \mathrm{Mg} \sim \mathrm{Fe} 2+\sim$ $\mathrm{Mn} \sim \mathrm{Ni} \sim \mathrm{Ca}$ (low ionic potential) $<\sim \mathrm{Na} \sim \mathrm{K}$ (lowest ionic potential) (Buggle et al., 2011). Figure 4 shows an ionic potential diagram with cations falling into different solubility regimes (Mitton, 1970). Buggle et al. (2011) considered Al and Na to be the best reference immobile and mobile elements, respectively.

Due to cracks and holes present in the chondrules (a possible byproduct of bleaching), the total weight percent values of some samples are lower than 100\%. By normalizing the values to $100 \%$ and taking the ratios of rim to core values this problem is removed and thus allows for a reasonable assessment of any mobilization or bleaching effect. The bulk accumulated data are shown in Table 2 which was used to calculate the bulk rim/core ratios for each element in each chondrule.

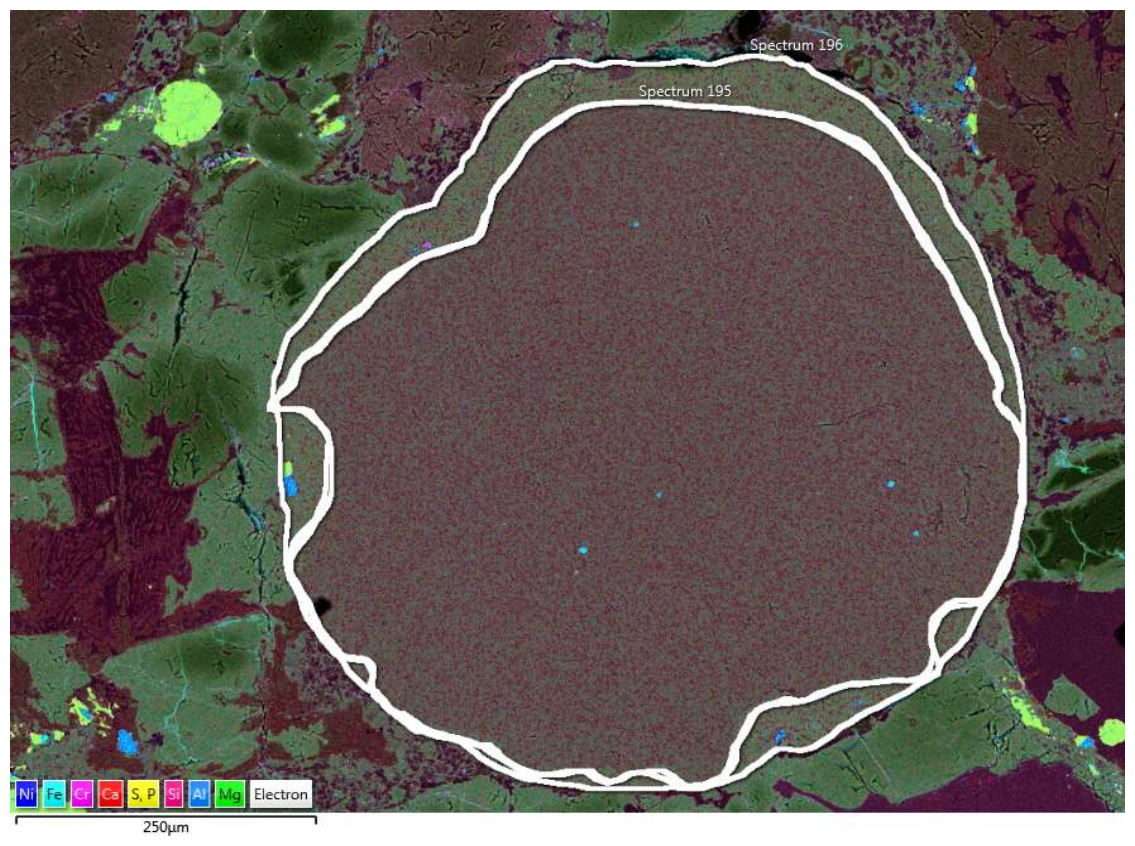

Figure 3: EDS Map of Bleached Chondrule 4 (0091-3). Spectrum sample 196 represents the bulk rim chemical measurement and spectrum sample 195 represent the bulk core chemical measurement. A clearly defined phase difference can be seen between core and rim as the rim is less silicon rich. 


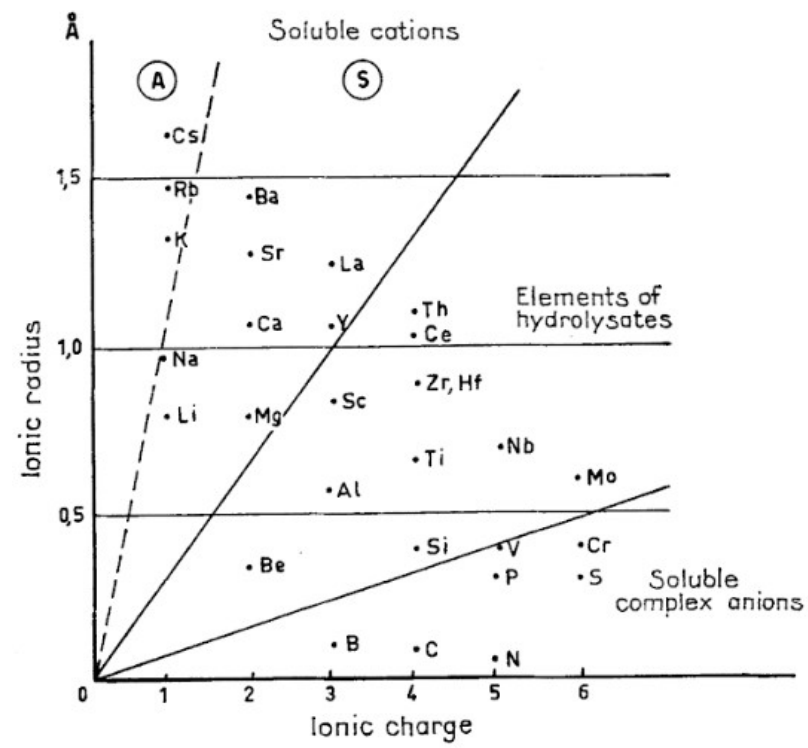

FIG. 6. - Distribution of elements according to ionic potential (after GoLDSCHMIDT, 1954, modified to distinguish the Antistokes - A- and Stokes $-S$ - soluble cations).

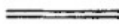

Figure 4: Ionic potential ranges of elements and how this affects solubility. The least soluble elements are the elements contained in the range of the hydrolysates. This diagram was obtained from the Geology of Clays (Millot, 1970). 
Table 2: Oxide weight percent of key elements needed to evaluate Grossman et al. 's hypothesis. Normalized oxide percentages are indicated with an *.

\begin{tabular}{|c|c|c|c|c|c|c|c|c|c|c|c|c|c|c|c|}
\hline & $\mathrm{Na}_{2} \mathrm{O}$ & $\mathrm{MgO}$ & $\mathrm{Al}_{2} \mathrm{O}_{3}$ & $\mathrm{SiO}_{2}$ & $\mathrm{P}_{2} \mathrm{O}_{5}$ & $\mathrm{Cl}$ & $\mathrm{K}_{2} \mathrm{O}$ & $\mathrm{CaO}$ & $\mathrm{TiO}_{2}$ & $\mathrm{Cr}_{2} \mathrm{O}_{3}$ & $\mathrm{MnO}$ & $\mathrm{FeO}$ & $\mathrm{S}$ & $\mathrm{Ni}$ & Total \\
\hline \multicolumn{16}{|c|}{ Chondrule 1 (0091-3) } \\
\hline Bulk Rim Ox\% & 0.12 & 29.52 & 0.89 & 36.90 & 0.00 & 0.00 & 0.06 & 0.86 & 0.11 & 0.83 & 0.46 & 27.51 & 0.01 & 0.22 & 97.5 \\
\hline Bulk Core Ox\% & 0.78 & 26.32 & 2.69 & 44.17 & 0.00 & 0.00 & 0.81 & 3.36 & 0.13 & 0.63 & 0.36 & 19.18 & 0.01 & 0.12 & 98.6 \\
\hline Bulk Rim Ox\%* & 0.12 & 30.28 & 0.91 & 37.85 & 0.00 & 0.00 & 0.06 & 0.88 & 0.11 & 0.85 & 0.47 & 28.22 & 0.01 & 0.23 & 100.0 \\
\hline Bulk Core Ox\%* & 0.79 & 26.70 & 2.73 & 44.82 & 0.00 & 0.00 & 0.82 & 3.41 & 0.13 & 0.64 & 0.37 & 19.46 & 0.01 & 0.12 & 100.0 \\
\hline \multicolumn{16}{|c|}{ Chondrule 2 (0091-3) } \\
\hline Bulk Rim Ox\% & 0.53 & 23.54 & 1.44 & 51.25 & 0.00 & 0.00 & 0.10 & 1.39 & 0.07 & 0.71 & 0.54 & 13.36 & 0.00 & 0.04 & 93.0 \\
\hline Bulk Core Ox\% & 0.68 & 22.49 & 1.86 & 56.19 & 0.00 & 0.00 & 0.18 & 1.25 & 0.09 & 0.73 & 0.49 & 10.90 & 0.00 & 0.03 & 94.9 \\
\hline Bulk Rim Ox\%* & 0.57 & 25.32 & 1.55 & 55.12 & 0.00 & 0.00 & 0.11 & 1.49 & 0.08 & 0.76 & 0.58 & 14.37 & 0.00 & 0.04 & 100.0 \\
\hline Bulk Core Ox\%* & 0.72 & 23.70 & 1.96 & 59.21 & 0.00 & 0.00 & 0.19 & 1.32 & 0.09 & 0.77 & 0.52 & 11.49 & 0.00 & 0.03 & 100.0 \\
\hline \multicolumn{16}{|c|}{ Chondrule 3 (0091-3) } \\
\hline Bulk Rim Ox\% & 0.43 & 27.33 & 0.73 & 38.62 & 0.04 & 0.00 & 0.07 & 1.93 & 0.09 & 0.71 & 0.50 & 24.76 & 0.03 & 0.08 & 95.3 \\
\hline Bulk Core Ox\% & 1.03 & 23.06 & 2.12 & 44.14 & 0.00 & 0.00 & 0.30 & 3.38 & 0.10 & 0.64 & 0.48 & 20.61 & 0.12 & 0.06 & 96.0 \\
\hline Bulk Rim Ox\%* & 0.45 & 28.67 & 0.77 & 40.51 & 0.04 & 0.00 & 0.07 & 2.02 & 0.09 & 0.74 & 0.52 & 25.97 & 0.03 & 0.08 & 100.0 \\
\hline Bulk Core Ox\%* & 1.07 & 24.01 & 2.21 & 45.96 & 0.00 & 0.00 & 0.31 & 3.52 & 0.10 & 0.67 & 0.50 & 21.46 & 0.12 & 0.06 & 100.0 \\
\hline \multicolumn{16}{|c|}{ Chondrule 4 (0091-3) } \\
\hline Bulk Rim Ox\% & 0.54 & 26.04 & 1.96 & 38.99 & 0.17 & 0.00 & 0.14 & 2.85 & 0.26 & 0.75 & 0.46 & 24.80 & 0.10 & 0.58 & 97.6 \\
\hline Bulk Core Ox\% & 0.93 & 21.61 & 3.48 & 43.66 & 0.08 & 0.00 & 1.09 & 2.74 & 0.19 & 0.57 & 0.41 & 20.57 & 0.00 & 0.07 & 95.4 \\
\hline Bulk Rim Ox\%* & 0.55 & 26.67 & 2.01 & 39.94 & 0.17 & 0.00 & 0.14 & 2.92 & 0.27 & 0.77 & 0.47 & 25.40 & 0.10 & 0.59 & 100.0 \\
\hline Bulk Core Ox\%* & 0.97 & 22.65 & 3.65 & 45.77 & 0.08 & 0.00 & 1.14 & 2.87 & 0.20 & 0.60 & 0.43 & 21.56 & 0.00 & 0.07 & 100.0 \\
\hline \multicolumn{16}{|c|}{ Chondrule 5 (0091-3) } \\
\hline Bulk Rim Ox\% & 0.40 & 22.04 & 0.94 & 47.90 & 0.00 & 0.00 & 0.09 & 1.46 & 0.09 & 0.67 & 0.62 & 19.71 & 0.04 & 0.23 & 94.2 \\
\hline Bulk Core Ox\% & 0.84 & 20.18 & 1.72 & 48.87 & 0.00 & 0.00 & 0.10 & 1.69 & 0.08 & 0.66 & 0.58 & 19.15 & 0.20 & 0.28 & 94.3 \\
\hline Bulk Rim Ox\%* & 0.42 & 23.40 & 1.00 & 50.85 & 0.00 & 0.00 & 0.10 & 1.55 & 0.10 & 0.71 & 0.66 & 20.93 & 0.04 & 0.24 & 100.0 \\
\hline Bulk Core Ox\%* & 0.89 & 21.39 & 1.82 & 51.80 & 0.00 & 0.00 & 0.11 & 1.79 & 0.08 & 0.70 & 0.61 & 20.30 & 0.21 & 0.30 & 100.0 \\
\hline \multicolumn{16}{|c|}{ Chondrule 6 (0781-A) } \\
\hline Bulk Rim Ox\% & 0.90 & 20.01 & 1.44 & 42.18 & 0.00 & 0.00 & 0.05 & 2.19 & 0.10 & 0.69 & 0.57 & 20.37 & 0.06 & 0.00 & 88.6 \\
\hline Bulk Core Ox\% & 0.21 & 20.50 & 0.21 & 33.02 & 0.00 & 0.00 & 0.00 & 0.92 & 0.07 & 0.67 & 0.50 & 34.21 & 0.03 & 0.00 & 90.3 \\
\hline Bulk Rim Ox\%* & 1.02 & 22.59 & 1.63 & 47.63 & 0.00 & 0.00 & 0.06 & 2.47 & 0.11 & 0.78 & 0.64 & 23.00 & 0.07 & 0.00 & 100.0 \\
\hline Bulk Core Ox\%* & 0.23 & 22.69 & 0.23 & 36.55 & 0.00 & 0.00 & 0.00 & 1.02 & 0.08 & 0.74 & 0.55 & 37.87 & 0.03 & 0.00 & 100.0 \\
\hline \multicolumn{16}{|c|}{ Chondrule 7 (0781-A) } \\
\hline Bulk Rim Ox\% & 0.55 & 23.14 & 0.80 & 46.18 & 0.00 & 0.00 & 0.00 & 1.25 & 0.07 & 0.67 & 0.78 & 13.21 & 0.12 & 0.07 & 86.8 \\
\hline Bulk Core Ox\% & 1.49 & 21.01 & 2.13 & 53.31 & 0.00 & 0.00 & 0.00 & 1.70 & 0.09 & 0.71 & 0.89 & 12.79 & 0.23 & 0.00 & 94.3 \\
\hline Bulk Rim Ox\%* & 0.63 & 26.65 & 0.92 & 53.18 & 0.00 & 0.00 & 0.00 & 1.44 & 0.08 & 0.77 & 0.90 & 15.21 & 0.14 & 0.08 & 100.0 \\
\hline Bulk Core Ox\%* & 1.58 & 22.27 & 2.26 & 56.51 & 0.00 & 0.00 & 0.00 & 1.80 & 0.10 & 0.75 & 0.94 & 13.56 & 0.24 & 0.00 & 100.0 \\
\hline \multicolumn{16}{|c|}{ Chondrule 8 (0781-A) } \\
\hline Bulk Rim Ox\% & 0.66 & 20.16 & 1.06 & 42.04 & 0.03 & 0.00 & 0.00 & 1.27 & 0.04 & 0.56 & 0.51 & 21.15 & 1.74 & 0.43 & 89.7 \\
\hline Bulk Core Ox\% & 1.25 & 21.34 & 2.05 & 48.62 & 0.09 & 0.00 & 0.00 & 1.69 & 0.07 & 0.64 & 0.59 & 18.24 & 1.23 & 0.13 & 96.0 \\
\hline Bulk Rim Ox\%* & 0.74 & 22.48 & 1.18 & 46.88 & 0.03 & 0.00 & 0.00 & 1.42 & 0.04 & 0.62 & 0.57 & 23.59 & 1.94 & 0.48 & 100.0 \\
\hline Bulk Core Ox\%* & 1.30 & 22.24 & 2.14 & 50.67 & 0.09 & 0.00 & 0.00 & 1.76 & 0.07 & 0.67 & 0.61 & 19.01 & 1.28 & 0.14 & 100.0 \\
\hline \multicolumn{16}{|c|}{ Chondrule 9 (0781-A) } \\
\hline Bulk Rim Ox\% & 0.82 & 18.77 & 1.09 & 39.20 & 0.03 & 0.00 & 0.01 & 1.28 & 0.08 & 0.67 & 0.60 & 24.36 & 0.17 & 0.00 & 87.1 \\
\hline Bulk Core Ox\% & 1.06 & 18.99 & 1.44 & 50.45 & 0.04 & 0.00 & 0.00 & 1.62 & 0.07 & 0.69 & 0.70 & 20.45 & 0.20 & 0.00 & 95.7 \\
\hline Bulk Rim Ox\%* & 0.94 & 21.56 & 1.25 & 45.03 & 0.03 & 0.00 & 0.01 & 1.47 & 0.09 & 0.77 & 0.69 & 27.98 & 0.20 & 0.00 & 100.0 \\
\hline Bulk Core Ox\%* & 1.11 & 19.84 & 1.50 & 52.72 & 0.04 & 0.00 & 0.00 & 1.69 & 0.07 & 0.72 & 0.73 & 21.37 & 0.21 & 0.00 & 100.0 \\
\hline
\end{tabular}

Subset Analysis:

Subset were obtained of rim and core areas for comparison to the bulk analyses. Ideally, the subsets avoid interference of unwanted grains or weathering byproducts and were taken on 
distinct minerals or regions of the mesostasis of the chondrule [Figure 5]. In some cases, the spectra were taken on similar multiphase assemblages in both the core and rim [Figure 6]. For each subset analysis, the subset sample number for the rim was $n=5$ as well as for the core. Each subset was normalized to 100 . Statistical analysis of these subset data points was computed by first finding the mean and standard deviation of each data pool (rim and core). The standard error of the mean was then calculated by using the standard deviation and dividing it by the n number of samples in the data pool. This allowed for the propagated error to be calculated for the ratios calculated for each element. The propagation of error was calculated by using the equation below:

$$
\partial R=R * \sqrt{\frac{\partial x^{2}}{x}+\frac{\partial y^{2}}{y}}
$$

\section{Equation 1}

where $\partial R$ is the propagated error, $\partial x$ is the standard error of the mean of the rim data pool, $\partial y$ is the standard error of the mean of the core data pool, $x$ is the mean of the rim data pool, $y$ is the mean of the core data pool, and $\mathrm{R}$ is the ratio of the rim over the core for a specific element. This was applied to all ratios and used to help determine which ratios where significant. The subset data was then compared directly to the bulk data. The average oxide $\%$ of the elements for each chondrule is shown in Table 3 which includes the standard deviation of each data pool as the error of the average.

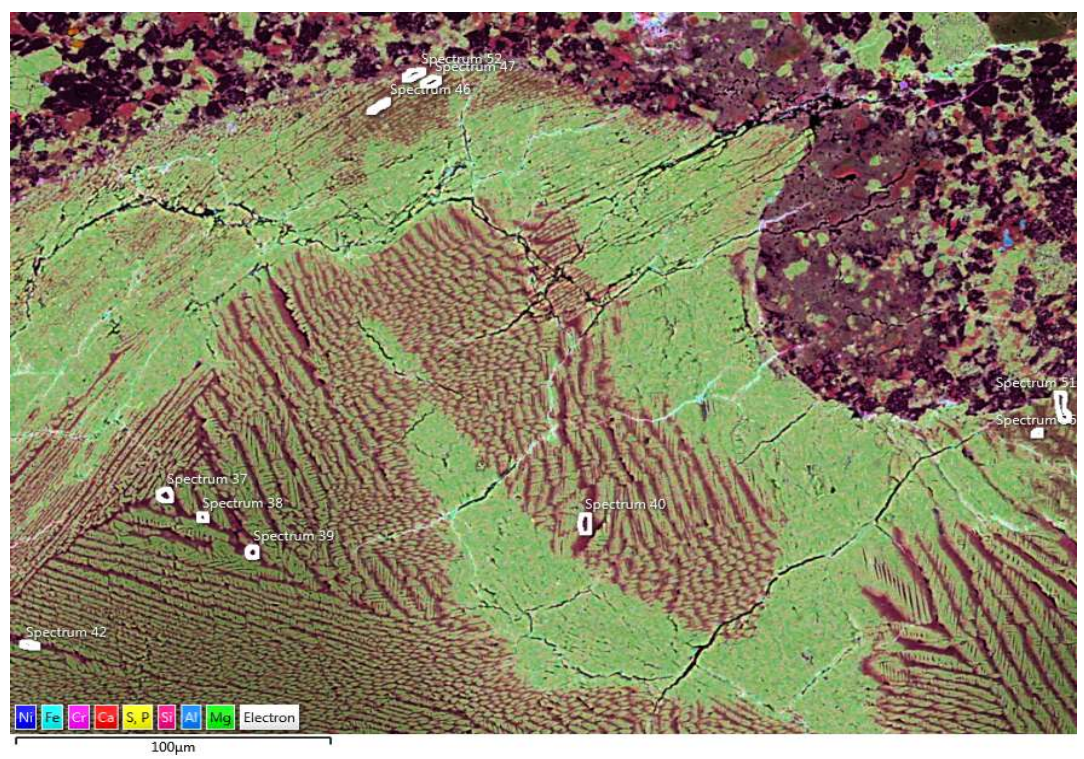

Figure 5: Subset data points of clear specific mesostasis phases seen in both core and rim of Chondrule 1. 


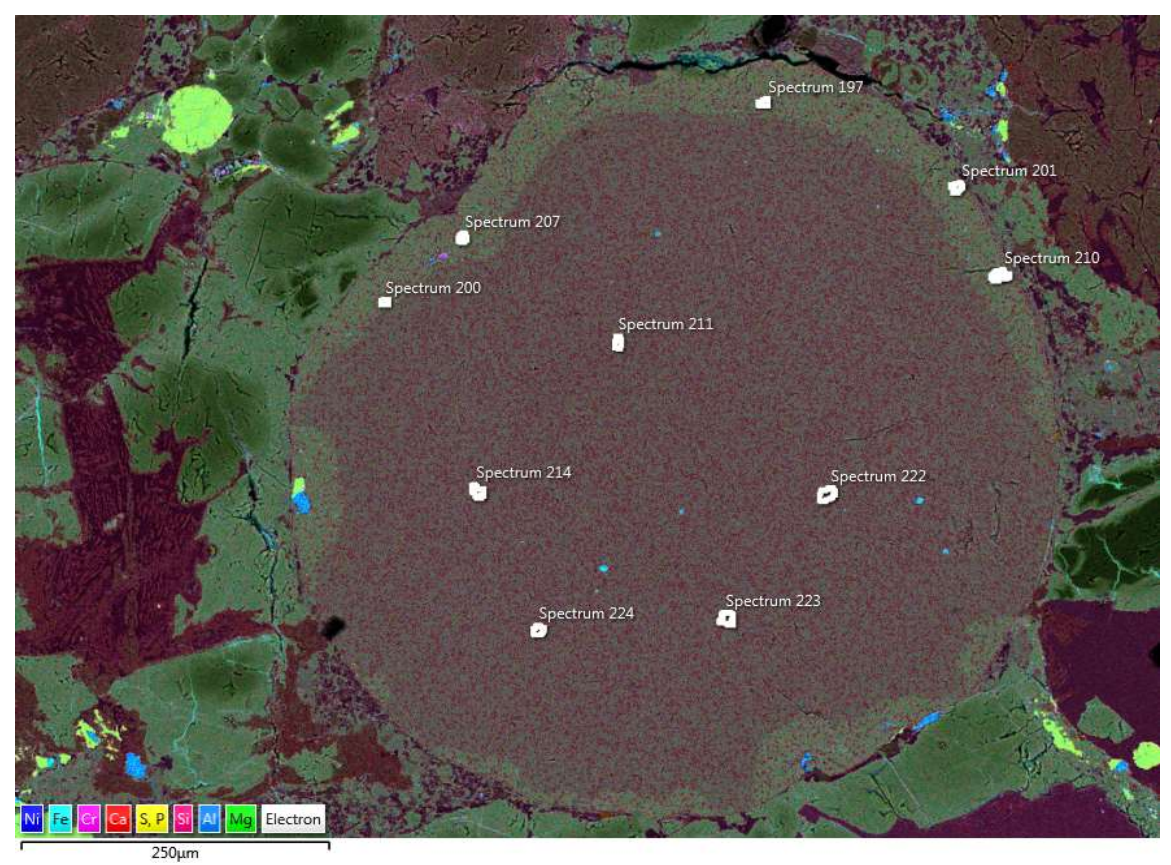

Figure 6: Subset data points of ambiguous mineral phases due to very small grain size seen in both core and rim of Chondrule 4.

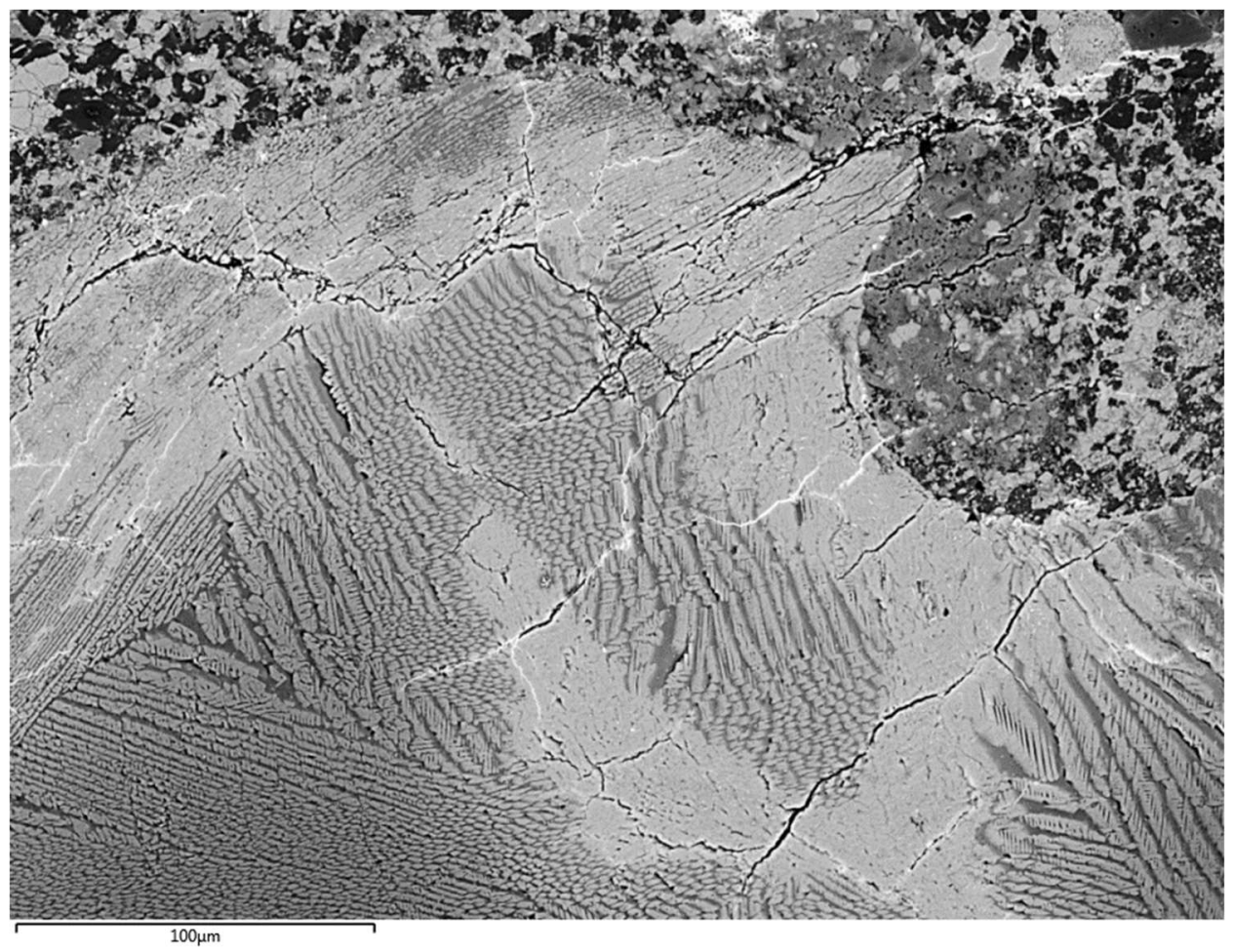

Figure 7: BSE image of chondrule 1. Black voids can be seen throughout the chondrule and were used to determine the porosity difference between core and rim. 
Table 3: Mean oxide percentages of subset data pools for each element of interest. An error value for each element was determined by the standard deviation of the sample pool. The total is the sum of the mean values of each element. Normalized oxide percentages are indicated with an *.

\begin{tabular}{|c|c|c|c|c|c|c|c|c|c|c|c|c|c|c|c|}
\hline & $\mathrm{Na}_{2} \mathrm{O}$ & $\mathrm{MgO}$ & $\mathrm{Al}_{2} \mathrm{O}_{3}$ & $\mathrm{SiO}_{2}$ & $\mathrm{P}_{2} \mathrm{O}_{5}$ & $\mathrm{Cl}$ & $\mathrm{K}_{2} \mathrm{O}$ & $\mathrm{CaO}$ & $\mathrm{TiO}_{2}$ & $\mathrm{Cr}_{2} \mathrm{O}_{3}$ & $\mathrm{MnO}$ & $\mathrm{FeO}$ & $\mathrm{s}$ & $\mathrm{Ni}$ & Total \\
\hline \multicolumn{16}{|c|}{ Chondrule 1 (0091-3) } \\
\hline Subset Rim Ox\% & $0.39 \pm 0.41$ & $22.4 \pm 2.54$ & $3.96 \pm 0.94$ & $51.19 \pm 1.93$ & $0 \pm 0$ & $0 \pm 0$ & $0 \pm 0$ & $7.79 \pm 2.68$ & $0.11 \pm 0.23$ & $1.71 \pm 0.29$ & $0.51 \pm 0.77$ & $10.94 \pm 1.78$ & $0.16 \pm 0.24$ & $0.7 \pm 0.66$ & 99.9 \\
\hline Subset Core Ox\% & $2.84 \pm 0.43$ & $10.13 \pm 2.72$ & $7.82 \pm 0.7$ & $60.43 \pm 4.05$ & $0 \pm 0$ & $0 \pm 0$ & $3.6 \pm 1.31$ & $8.11 \pm 3.23$ & $0.39 \pm 0.56$ & $1.13 \pm 0.78$ & $0 \pm 0$ & $4.36 \pm 1.77$ & $0.12 \pm 0.16$ & $0 \pm 0$ & 98.9 \\
\hline Subset Rim Ox\%** & $0.4 \pm 0.41$ & $22.44 \pm 2.58$ & $3.97 \pm 0.94$ & $51.27 \pm 1.46$ & $0 \pm 0$ & $0 \pm 0$ & $0 \pm 0$ & $7.81 \pm 2.72$ & $0.11 \pm 0.24$ & $1.71 \pm 0.27$ & $0.51 \pm 0.76$ & $10.95 \pm 1.77$ & $0.16 \pm 0.25$ & $0.69 \pm 0.65$ & 100.0 \\
\hline Subset Core Ox\%* & $2.87 \pm 0.42$ & $10.24 \pm 2.78$ & $7.9 \pm 0.7$ & $61.05 \pm 4.06$ & $0 \pm 0$ & $0 \pm 0$ & $3.64 \pm 1.33$ & $8.18 \pm 3.23$ & $0.4 \pm 0.57$ & $1.14 \pm 0.79$ & $0 \pm 0$ & $4.4 \pm 1.79$ & $0.12 \pm 0.16$ & $0 \pm 0$ & 99.9 \\
\hline \multicolumn{16}{|l|}{ Chondrule 2 (0091-3) } \\
\hline Subset Rim Ox\% & $0.07 \pm 0.15$ & $25.72 \pm 0.95$ & $0.86 \pm 0.28$ & $54.29 \pm 1.07$ & $0 \pm 0$ & $0 \pm 0$ & $0.04 \pm 0.08$ & $1.89 \pm 0.59$ & $0 \pm 0$ & $0.63 \pm 0.26$ & $0.6 \pm 0.2$ & $15 \pm 1.06$ & $0 \pm 0$ & $0.09 \pm 0.19$ & 99.2 \\
\hline Subset Core Ox\% & $0.8 \pm 0.51$ & $26.39 \pm 1.8$ & $1.55 \pm 0.44$ & $56.04 \pm 1.18$ & $0 \pm 0$ & $0 \pm 0$ & $0.13 \pm 0.18$ & $1.14 \pm 0.43$ & $0 \pm 0$ & $0.9 \pm 0.29$ & $0.49 \pm 0.48$ & $12.16 \pm 1.23$ & $0.06 \pm 0.13$ & $0.11 \pm 0.25$ & 99.8 \\
\hline Subset Rim Ox\%* & $0.07 \pm 0.15$ & $25.92 \pm 0.61$ & $0.87 \pm 0.29$ & $54.74 \pm 0.93$ & $0 \pm 0$ & $0 \pm 0$ & $0.04 \pm 0.08$ & $1.91 \pm 0.6$ & $0 \pm 0$ & $0.64 \pm 0.27$ & $0.6 \pm 0.2$ & $15.11 \pm 0.83$ & $0 \pm 0$ & $0.09 \pm 0.19$ & 100.0 \\
\hline Subset Core Ox\%* & $0.8 \pm 0.5$ & $26.45 \pm 1.73$ & $1.56 \pm 0.44$ & $56.18 \pm 1.33$ & $0 \pm 0$ & $0 \pm 0$ & $0.13 \pm 0.18$ & $1.14 \pm 0.44$ & $0 \pm 0$ & $0.9 \pm 0.29$ & $0.49 \pm 0.48$ & $12.18 \pm 1.11$ & $0.06 \pm 0.13$ & $0.11 \pm 0.25$ & 100.0 \\
\hline \multicolumn{16}{|l|}{ Chondrule 3 (0091-3) } \\
\hline Subset Rim Ox\% & $1.1 \pm 1.17$ & $21.14 \pm 4.34$ & $1.85 \pm 2.59$ & $44.45 \pm 1.65$ & $0.58 \pm 0.55$ & $0 \pm 0$ & $0 \pm 0$ & $4.77 \pm 2.94$ & $0 \pm 0$ & $0.8 \pm 0.59$ & $0.23 \pm 0.32$ & $18.62 \pm 3.06$ & $0.08 \pm 0.18$ & $0.09 \pm 0.2$ & 93.7 \\
\hline Subset Core Ox\% & $2.75 \pm 0.76$ & $11.05 \pm 2.53$ & $5.16 \pm 0.27$ & $57.23 \pm 3.58$ & $0.2 \pm 0.45$ & $0 \pm 0$ & $0.25 \pm 0.24$ & $6.18 \pm 0.34$ & $0 \pm 0$ & $0.55 \pm 0.57$ & $0.35 \pm 0.51$ & $9.97 \pm 2.04$ & $0.05 \pm 0.1$ & $0 \pm 0$ & 93.7 \\
\hline \multicolumn{16}{|l|}{ Chondrule 4 (0091-3) } \\
\hline Subset Rim Ox\% & $0.5 \pm 0.68$ & $23.8 \pm 1.72$ & $1.34 \pm 1.17$ & $44.73 \pm 1.48$ & $0 \pm 0$ & $0 \pm 0$ & $0.17 \pm 0.25$ & $4.71 \pm 0.85$ & $0.19 \pm 0.43$ & $0.76 \pm 0.7$ & $0.16 \pm 0.36$ & $20.76 \pm 0.68$ & $0 \pm 0$ & $0 \pm 0$ & 97.1 \\
\hline Subset Core Ox\% & $0.57 \pm 0.84$ & $20.83 \pm 0.74$ & $3 \pm 0.88$ & $46.24 \pm 1.79$ & $0 \pm 0$ & $0 \pm 0$ & $1.07 \pm 0.18$ & $3.5 \pm 0.25$ & $0 \pm 0$ & $0.57 \pm 0.8$ & $0.22 \pm 0.31$ & $19.09 \pm 0.99$ & $0 \pm 0$ & $0 \pm 0$ & 95.1 \\
\hline Subset Rim Ox\%* & $0.51 \pm 0.7$ & $24.5 \pm 1.75$ & $1.39 \pm 1.22$ & $46.05 \pm 1.51$ & $0 \pm 0$ & $0 \pm 0$ & $0.18 \pm 0.26$ & $4.84 \pm 0.84$ & $0.2 \pm 0.45$ & $0.79 \pm 0.72$ & $0.17 \pm 0.37$ & $21.38 \pm 0.68$ & $0 \pm 0$ & $0 \pm 0$ & 100.0 \\
\hline Subset Core Ox\%* & $0.6 \pm 0.88$ & $21.9 \pm 0.64$ & $3.16 \pm 0.94$ & $48.61 \pm 1.19$ & $0 \pm 0$ & $0 \pm 0$ & $1.12 \pm 0.19$ & $3.68 \pm 0.24$ & $0 \pm 0$ & $0.6 \pm 0.85$ & $0.24 \pm 0.33$ & $20.09 \pm 1.16$ & $0 \pm 0$ & $0 \pm 0$ & 100.0 \\
\hline \multicolumn{16}{|l|}{ Chondrule 5 (0091-3) } \\
\hline Subset Rim Ox\% & $0 \pm 0$ & $21.09 \pm 0.67$ & $0.2 \pm 0.44$ & $50.51 \pm 0.91$ & $0 \pm 0$ & $0 \pm 0$ & $0 \pm 0$ & $1.7 \pm 0.41$ & $0.13 \pm 0.28$ & $0.75 \pm 0.48$ & $0.55 \pm 0.52$ & $18.45 \pm 1.61$ & $0 \pm 0$ & $0.12 \pm 0.26$ & 93.5 \\
\hline Subset Core Ox\% & $0.73 \pm 0.67$ & $20.79 \pm 1.19$ & $1.18 \pm 1.23$ & $53.2 \pm 1.48$ & $0 \pm 0$ & $0 \pm 0$ & $0.12 \pm 0.28$ & $1.75 \pm 0.85$ & $0.5 \pm 0.7$ & $0.54 \pm 0.74$ & $0.25 \pm 0.56$ & $16.32 \pm 2.64$ & $0.09 \pm 0.19$ & $0.16 \pm 0.35$ & 95.6 \\
\hline Subset Rim Ox\%* & $0 \pm 0$ & $22.56 \pm 0.71$ & $0.21 \pm 0.47$ & $54.03 \pm 0.86$ & $0 \pm 0$ & $0 \pm 0$ & $0 \pm 0$ & $1.82 \pm 0.45$ & $0.14 \pm 0.3$ & $0.8 \pm 0.51$ & $0.59 \pm 0.56$ & $19.73 \pm 1.67$ & $0 \pm 0$ & $0.12 \pm 0.28$ & 100.0 \\
\hline Subset Core Ox\%* & $0.76 \pm 0.71$ & $21.69 \pm 0.77$ & $1.2 \pm 1.24$ & $55.58 \pm 2.48$ & $0 \pm 0$ & $0 \pm 0$ & $0.13 \pm 0.3$ & $1.84 \pm 0.92$ & $0.54 \pm 0.75$ & $0.57 \pm 0.79$ & $0.26 \pm 0.57$ & $16.99 \pm 2.29$ & $0.09 \pm 0.2$ & $0.15 \pm 0.35$ & 99.8 \\
\hline \multicolumn{16}{|c|}{ Chondrule 6 (0781-A) } \\
\hline Subset Rim Ox\% & $1.3 \pm 0.9$ & $20.63 \pm 1.18$ & $2.22 \pm 1.55$ & $44.33 \pm 0.56$ & $0 \pm 0$ & $0 \pm 0$ & $0.11 \pm 0.12$ & $2.36 \pm 0.39$ & $0.16 \pm 0.1$ & $0.8 \pm 0.05$ & $0.56 \pm 0.11$ & $21.36 \pm 1.23$ & $0.06 \pm 0.02$ & $0.02 \pm 0.04$ & 93.9 \\
\hline Subset Core Ox\% & $0.21 \pm 0.04$ & $21.27 \pm 0.25$ & $0.14 \pm 0.09$ & $32.65 \pm 0.78$ & $0 \pm 0$ & $0 \pm 0$ & $0 \pm 0$ & $0.46 \pm 0.17$ & $0.06 \pm 0.05$ & $0.71 \pm 0.05$ & $0.5 \pm 0.04$ & $36.92 \pm 0.53$ & $0.02 \pm 0.03$ & $0 \pm 0$ & 92.9 \\
\hline Subset Core Ox\%* & $0.22 \pm 0.04$ & $22.88 \pm 0.25$ & $0.15 \pm 0.1$ & $35.12 \pm 0.43$ & $0 \pm 0$ & $0 \pm 0$ & $0 \pm 0$ & $0.49 \pm 0.17$ & $0.06 \pm 0.06$ & $0.76 \pm 0.06$ & $0.54 \pm 0.05$ & $39.72 \pm 0.5$ & $0.02 \pm 0.03$ & $0 \pm 0$ & 100.0 \\
\hline \multicolumn{16}{|l|}{ Chondrule 7 (0781-A) } \\
\hline Subset Rim Ox\% & $0.07 \pm 0.1$ & $30.07 \pm 0.52$ & $0.15 \pm 0.21$ & $55.29 \pm 1.14$ & $0 \pm 0$ & $0 \pm 0$ & $0 \pm 0$ & $0.26 \pm 0.11$ & $0.03 \pm 0.06$ & $0.76 \pm 0.07$ & $0.81 \pm 0.15$ & $11.48 \pm 0.52$ & $0.03 \pm 0.07$ & $0 \pm 0$ & 99.0 \\
\hline Subset Core Ox\% & $0.23 \pm 0.1$ & $28.31 \pm 0.72$ & $0.28 \pm 0.22$ & $54.16 \pm 1.34$ & $0.03 \pm 0.06$ & $0 \pm 0$ & $0 \pm 0$ & $0.64 \pm 0.14$ & $0.04 \pm 0.06$ & $0.77 \pm 0.09$ & $0.76 \pm 0.23$ & $12.07 \pm 0.56$ & $0.04 \pm 0.06$ & $0.05 \pm 0.1$ & 97.4 \\
\hline Subset Rim Ox\%* & $0.07 \pm 0.1$ & $30.4 \pm 0.42$ & $0.15 \pm 0.21$ & $55.88 \pm 0.33$ & $0 \pm 0$ & $0 \pm 0$ & $0 \pm 0$ & $0.26 \pm 0.11$ & $0.03 \pm 0.06$ & $0.77 \pm 0.08$ & $0.82 \pm 0.15$ & $11.6 \pm 0.45$ & $0.03 \pm 0.07$ & $0 \pm 0$ & 100.0 \\
\hline Subset Core Ox\%* & $0.23 \pm 0.1$ & $29.08 \pm 0.69$ & $0.29 \pm 0.23$ & $55.62 \pm 0.21$ & $0.03 \pm 0.07$ & $0 \pm 0$ & $0 \pm 0$ & $0.66 \pm 0.15$ & $0.04 \pm 0.06$ & $0.79 \pm 0.08$ & $0.79 \pm 0.24$ & $12.39 \pm 0.31$ & $0.04 \pm 0.06$ & $0.05 \pm 0.1$ & 100.0 \\
\hline \multicolumn{16}{|c|}{ Chondrule 8 (0781-A) } \\
\hline Subset Rim Ox\% & $0.48 \pm 0.15$ & $23.47 \pm 0.56$ & $0.89 \pm 0.46$ & $49.85 \pm 1.68$ & $0 \pm 0$ & $0 \pm 0$ & $0.02 \pm 0.06$ & $1.61 \pm 0.32$ & $0.08 \pm 0.11$ & $0.64 \pm 0.24$ & $0.7 \pm 0.21$ & $19.58 \pm 2.41$ & $0.29 \pm 0.16$ & $0.39 \pm 0.39$ & 98.0 \\
\hline Subset Core Ox\% & $1.28 \pm 0.12$ & $22.31 \pm 0.77$ & $2.23 \pm 0.22$ & $51.64 \pm 1.66$ & $0.09 \pm 0.15$ & $0 \pm 0$ & $0 \pm 0$ & $1.83 \pm 0.26$ & $0.08 \pm 0.07$ & $0.68 \pm 0.04$ & $0.69 \pm 0.13$ & $16.78 \pm 0.77$ & $0.25 \pm 0.06$ & $0 \pm 0$ & 97.9 \\
\hline Subset Rim Ox\%* & $0.49 \pm 0.15$ & $23.95 \pm 0.18$ & $0.91 \pm 0.47$ & $50.87 \pm 1.61$ & $0 \pm 0$ & $0 \pm 0$ & $0.02 \pm 0.05$ & $1.64 \pm 0.33$ & $0.08 \pm 0.12$ & $0.65 \pm 0.23$ & $0.71 \pm 0.21$ & $19.97 \pm 2.31$ & $0.3 \pm 0.16$ & $0.4 \pm 0.4$ & 100.0 \\
\hline Subset Core Ox\%* & $1.31 \pm 0.1$ & $22.79 \pm 0.33$ & $2.28 \pm 0.24$ & $52.77 \pm 0.35$ & $0.09 \pm 0.15$ & $0 \pm 0$ & $0 \pm 0$ & $1.87 \pm 0.27$ & $0.08 \pm 0.07$ & $0.69 \pm 0.04$ & $0.7 \pm 0.12$ & $17.15 \pm 0.66$ & $0.26 \pm 0.06$ & $0 \pm 0$ & 100.0 \\
\hline \multicolumn{16}{|c|}{ Chondrule 9 (0781-A) } \\
\hline Subset Rim Ox\% & $0.64 \pm 0.07$ & $20.45 \pm 0.6$ & $0.92 \pm 0.24$ & $45.27 \pm 1.91$ & $0.03 \pm 0.07$ & $0 \pm 0$ & $0 \pm 0$ & $1.47 \pm 0.17$ & $0.12 \pm 0.07$ & $0.73 \pm 0.14$ & $0.69 \pm 0.07$ & $21.22 \pm 1.37$ & $0.02 \pm 0.03$ & $0 \pm 0$ & 91.6 \\
\hline Subset Core Ox\% & $1.06 \pm 0.08$ & $19.2 \pm 0.67$ & $1.47 \pm 0.1$ & $51.76 \pm 2.7$ & $0.03 \pm 0.05$ & $0 \pm 0$ & $0 \pm 0$ & $1.68 \pm 0.09$ & $0.09 \pm 0.02$ & $0.71 \pm 0.05$ & $0.72 \pm 0.03$ & $20.1 \pm 1.42$ & $0.18 \pm 0.03$ & $0 \pm 0$ & 97.0 \\
\hline Subset Rim Ox\%* & $0.7 \pm 0.08$ & $22.33 \pm 0.31$ & $1 \pm 0.28$ & $49.43 \pm 1.41$ & $0.03 \pm 0.08$ & $0 \pm 0$ & $0 \pm 0$ & $1.6 \pm 0.16$ & $0.13 \pm 0.08$ & $0.79 \pm 0.14$ & $0.75 \pm 0.07$ & $23.2 \pm 1.8$ & $0.02 \pm 0.03$ & $0 \pm 0$ & 100.0 \\
\hline Subset Core Ox\%* & $1.09 \pm 0.08$ & $19.79 \pm 0.32$ & $1.51 \pm 0.08$ & $53.34 \pm 1.7$ & $0.03 \pm 0.05$ & $0 \pm 0$ & $0 \pm 0$ & $1.73 \pm 0.12$ & $0.09 \pm 0.02$ & $0.73 \pm 0.05$ & $0.74 \pm 0.02$ & $20.75 \pm 1.91$ & $0.19 \pm 0.03$ & $0 \pm 0$ & 100.0 \\
\hline
\end{tabular}

\section{Porosity Test:}

A qualitative analysis was used to test porosity of the multiple chondrule samples as the porosity of each the bleached chondrules is a key component in understand the potential influence of 
aqueous alteration. Using BSE images obtained from the SEM, stark black regions in the images were determined to be open spaces/cracks. They were not considered to be atomically light elements as it can be determined from Figure 5 \& Figure 7 that the regions of open space where not characterized to have chemical compositions. Using these black voids as key identifiers for porosity, each sample was viewed, and it was determined if the core of the chondrule or the rim of the chondrule was more porous. Given that these objects are be three dimensional as a full grain, large cracks were considered to be part of the porosity seen within a specific region as the cracks would be throughout more of the grain than the thin section sample is able to justify.

\section{$\mathrm{FeO} \& \mathrm{Na}$ Content:}

The final procedural method was performed to see how $\mathrm{Fe} /(\mathrm{Fe}+\mathrm{Mg})$ content and $\mathrm{Na}$ content related to solubility. This was done by comparing the presumed least soluble element Al's ratio to both the $\mathrm{Fe} /(\mathrm{Fe}+\mathrm{Mg})$ ratios from core to rim as well as the $\mathrm{Na}$ ratio. Also, the oxide ratios of $\mathrm{Fe} /(\mathrm{Fe}+\mathrm{Mg})$ from rim to core were calculated from Grossman et al.'s (2000) data and then compared to the oxide ratios of the nine analyzed chondrules for this report. 


\section{Results:}

All of the false color maps for the chondrules obtained are shown in Figure 8 and Figure 9 below, for both NWA 11991 and Tieschitz. These maps were used to define the rims and core of each chondrule.
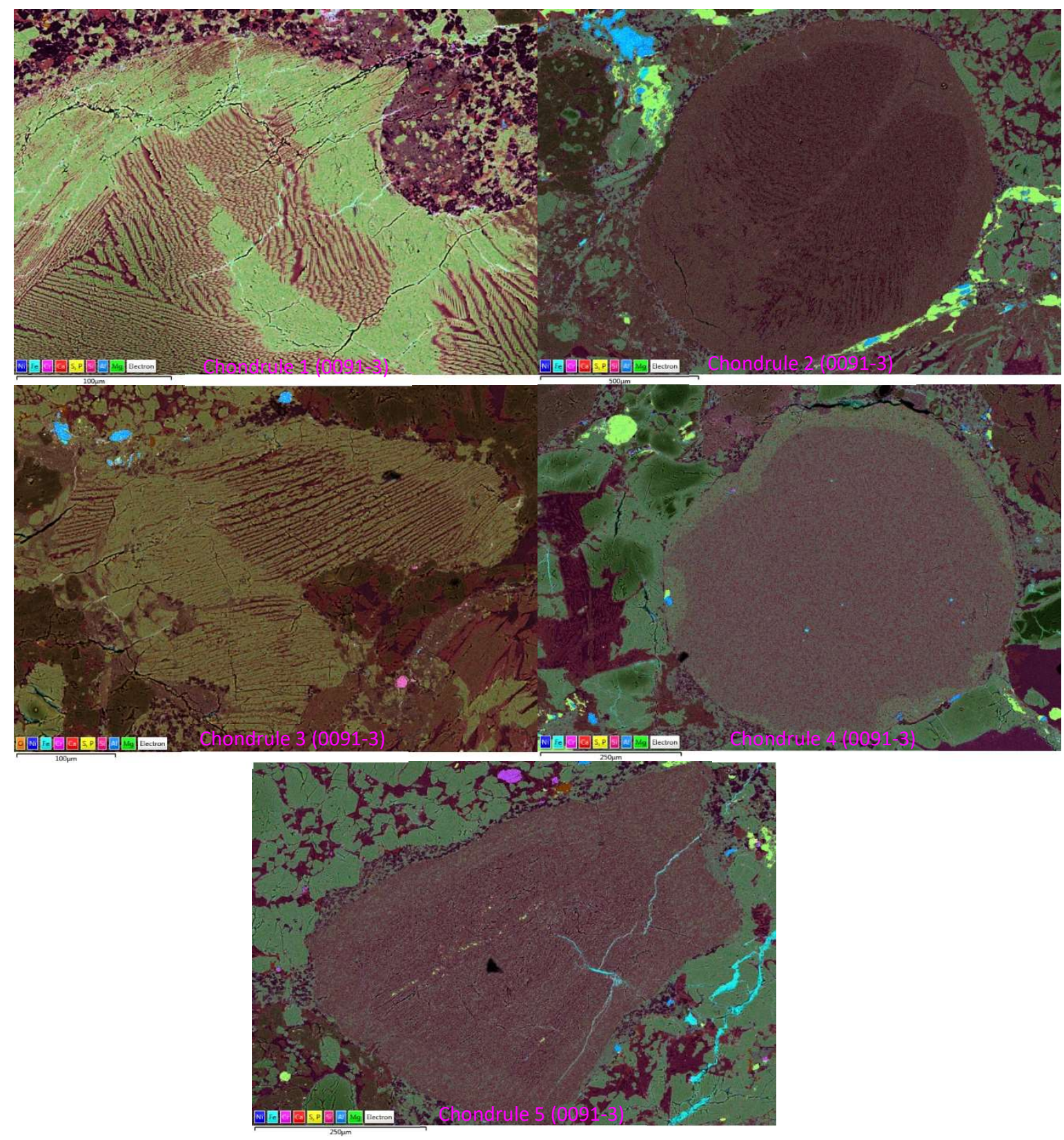

Figure 8: False color maps of each chondrule analyzed in NWA 11991, sample 0091-3. These maps were used to find clear rim to core boundaries. 

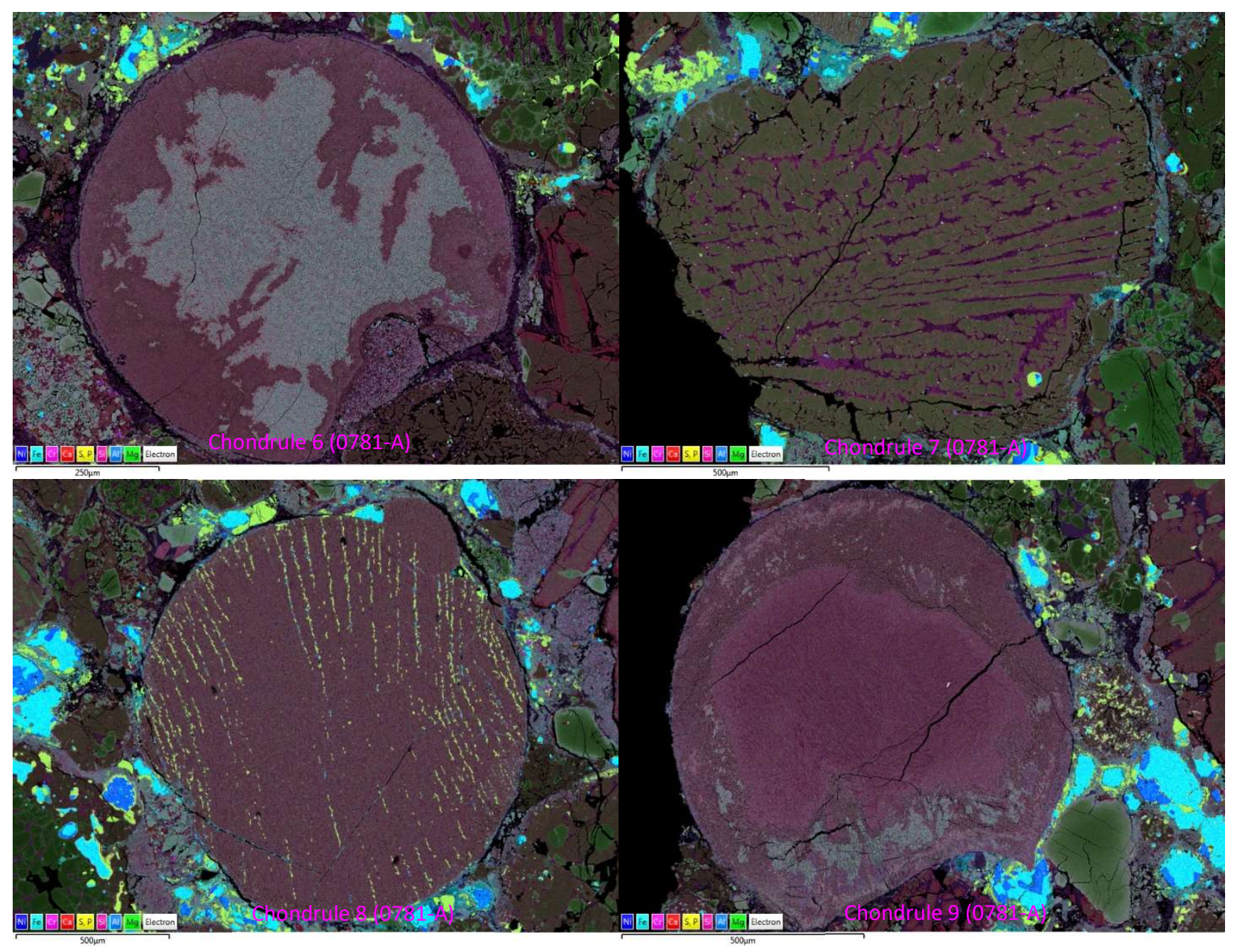

Figure 9: False color maps of each chondrule analyzed in Tieschitz, sample 0091-3. These maps were used to find clear rim to core boundaries.

Rim/core element concentration ratios for both large-area bulk extracts as well as average subsets are shown in Figure 10 (chondrules 1-9). All subset ratios with zero values were removed, and elements with zero values for both the bulk and subset ratios were removed from the plots. Only one data set included a subset ratio in which the value was greater than zero while the bulk value was found to be zero, which is seen for the phosphorous content of Chondrule 3 . Since the subset data was looking at a clearly defined phase, the mesostasis, this value was not removed but the significance of the $\mathrm{P}$ data for Chondrule 3 is not clear. Ni rim/core ratios were also removed from chondrules 4 and 8 , as subsets indicated zero values and the large extract data indicated caused by Ni-rich grains or terrestrial weathering. Chondrule 6 in Figure 10 is represented without the $\mathrm{Al}$ ratio as the $\mathrm{Al}$ ratio was off-scale high but this high ratio appears to be significant based on the subset data, and is shown on an expanded scale in Figure 11. 


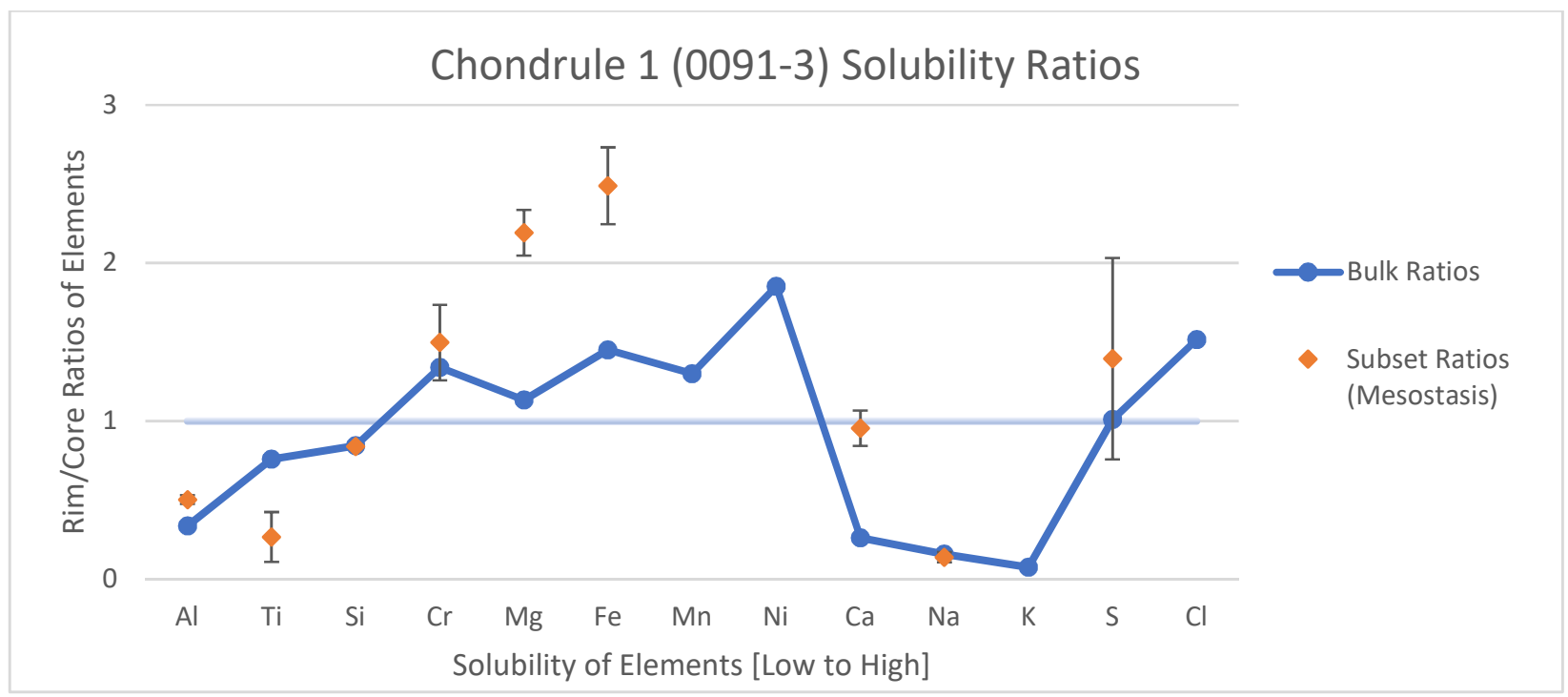

Chondrule 2 (0091-3) Solubility Ratios
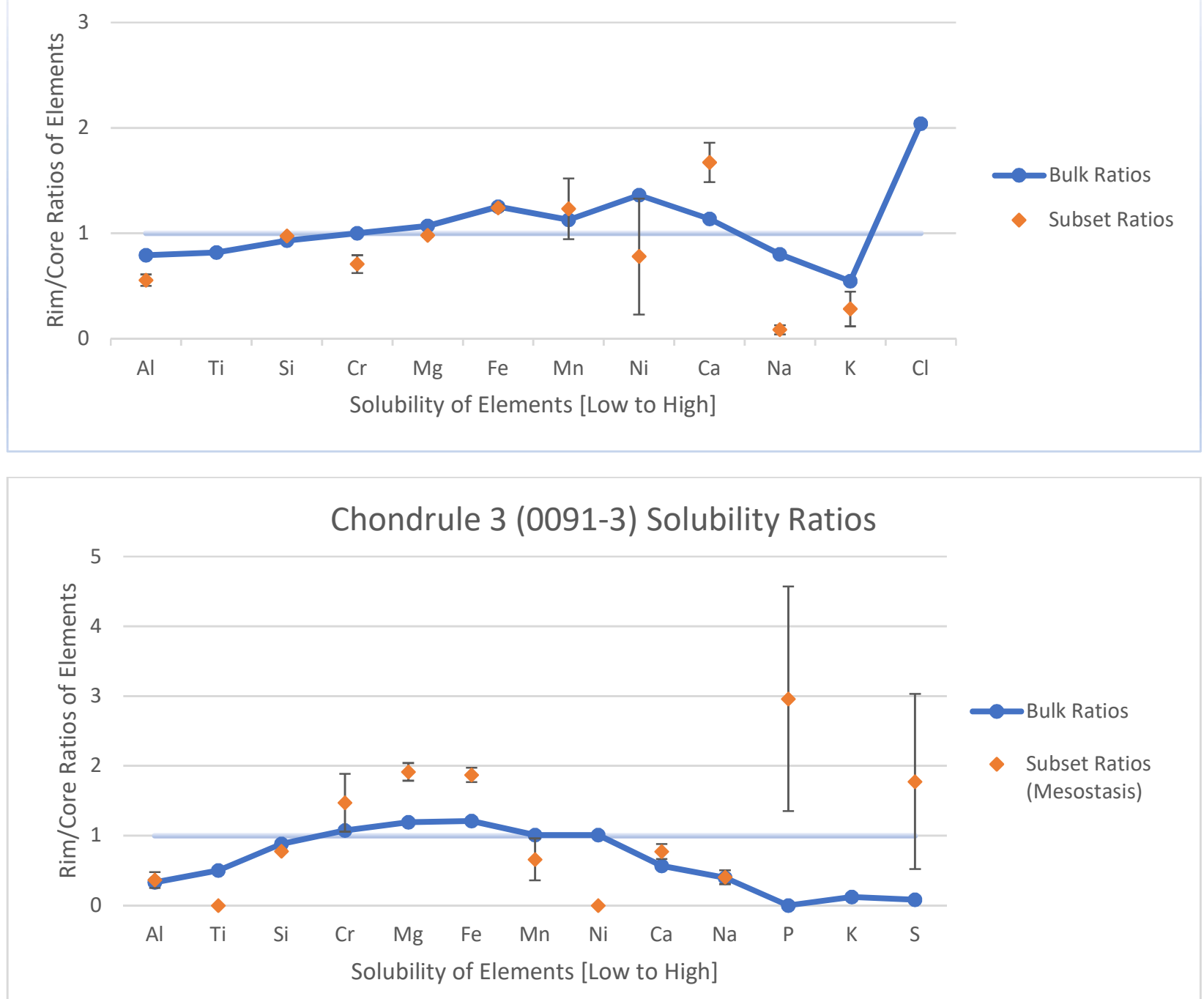


\section{Chondrule 4 (0091-3) Solubility Ratios}
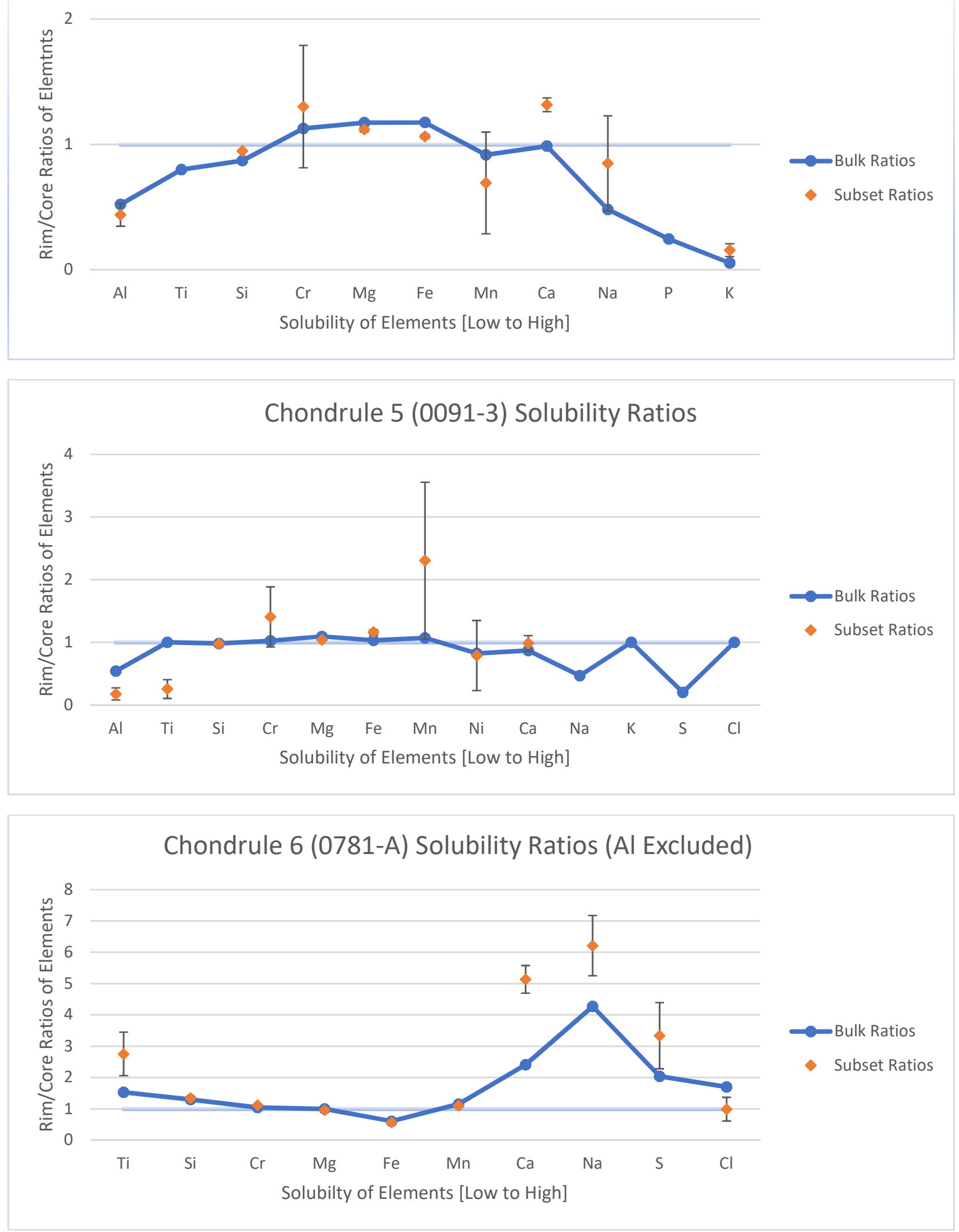

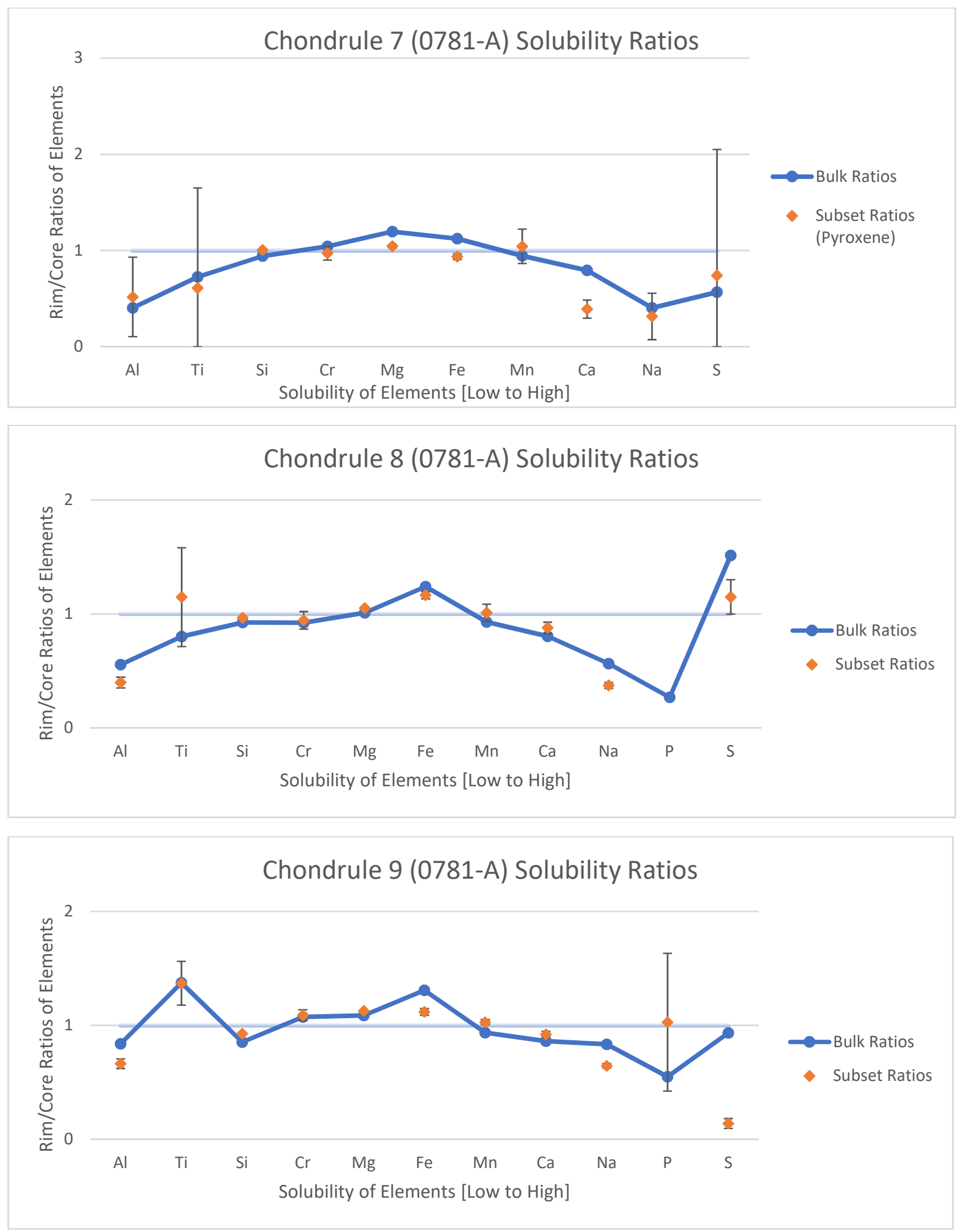

Figure 10: Bulk \& subset chemical results for all nine chondrules. The solubility of each element increases from left to right. A light line is shown at the value of 1, any ratio above this line present as a higher quantity in the rim. Below it is the element quantity is greater in the core. 


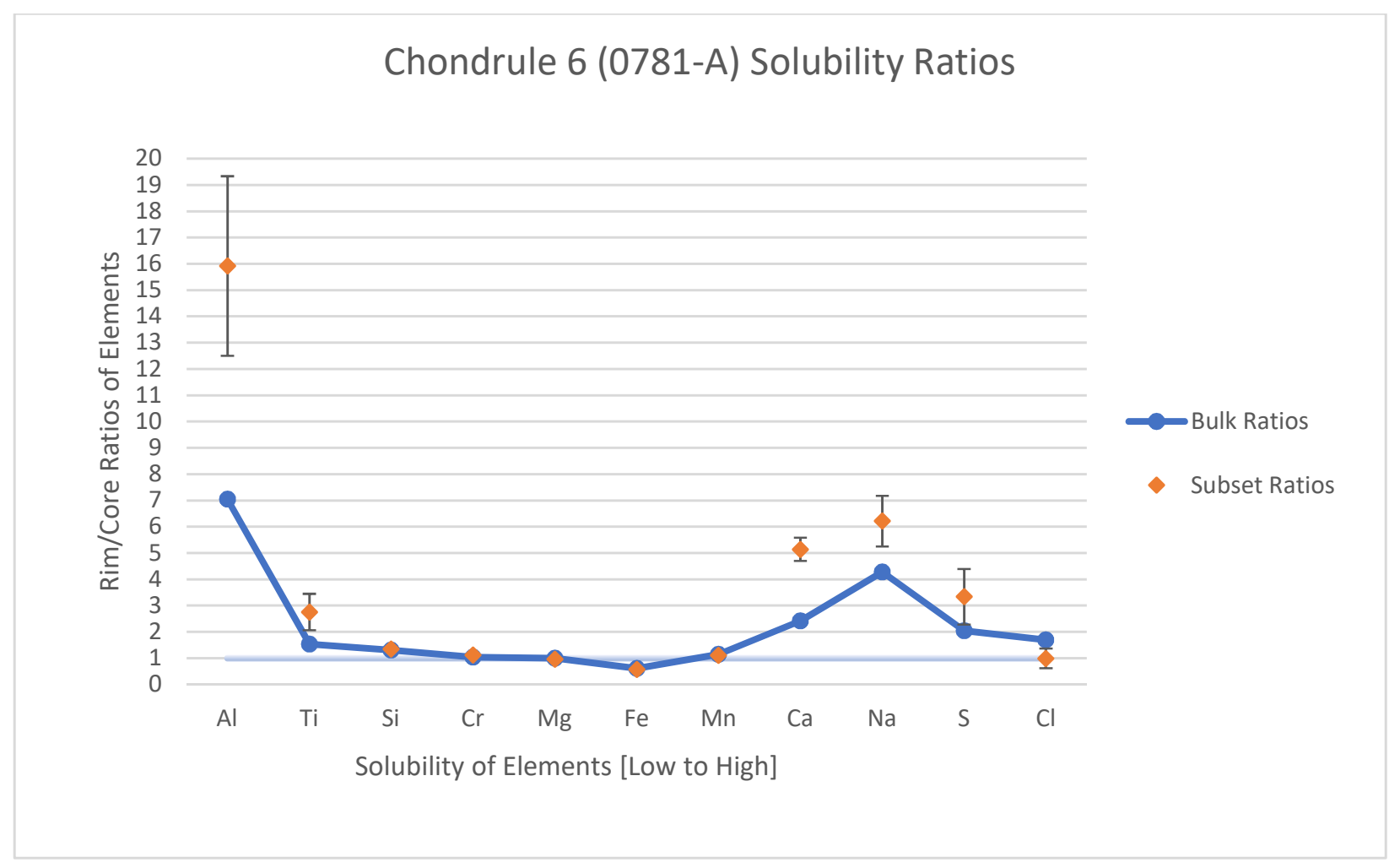

Figure 11: Bulk and subset chemical results including the Al ratio data. This abundance of Al is justified by the subset data.

Figure 12 combines all of the bulk ratios in one plot.

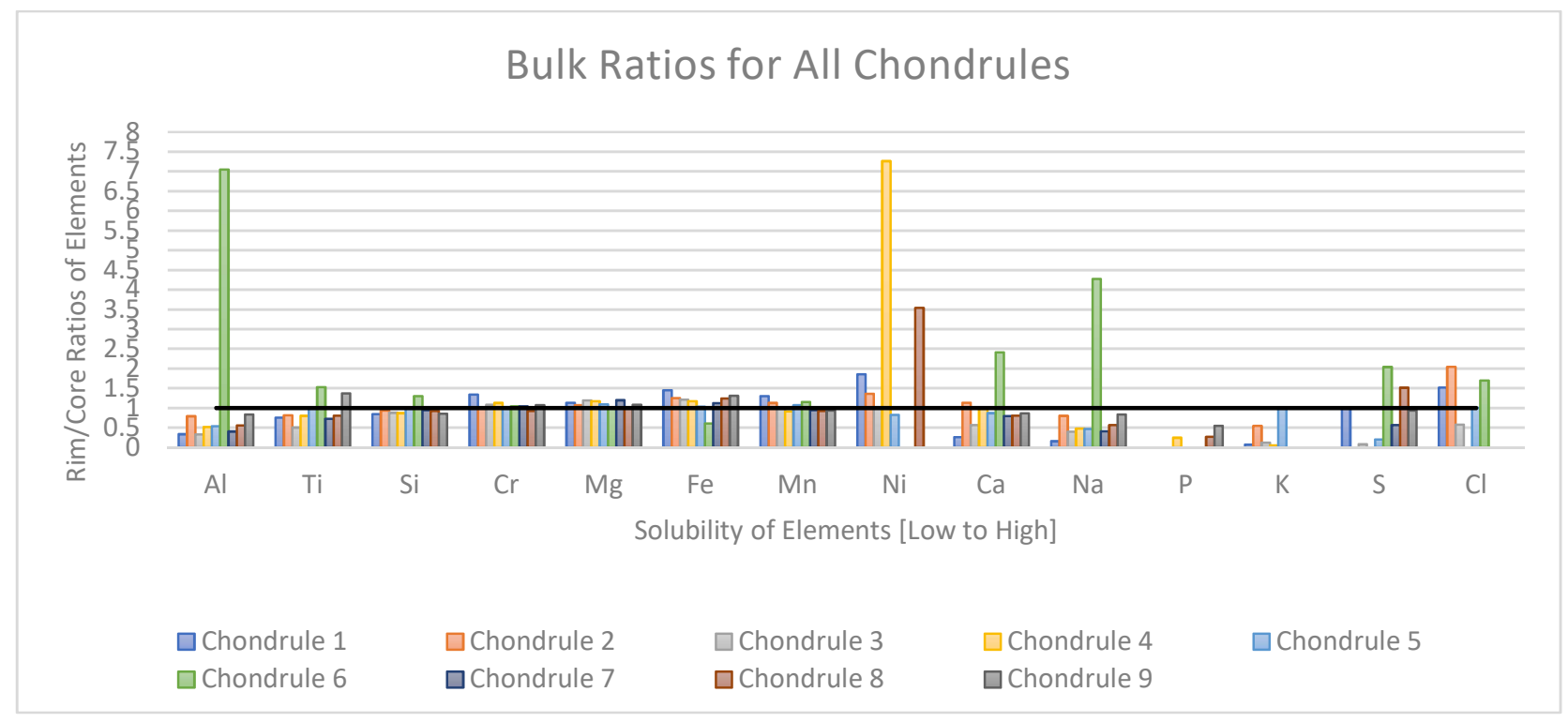

Figure 12: Total bulk chemical results of every chondrule. The black line is at a value of 1 to divide rim and core abundance of an element. 
To reduce the information found in Figure 12, four bulk chondrule ratios were plotted against each other in Figure 13. Due to Chondrule 7's optical representation, its porosity [Figure 14], and its chemical composition, it was determined that it fit the description of Grossman et al.'s bleached chondrules the most accurately of the nine chondrules. It was used as a reference point in which the chondrules that deviated from Grossman et al's description significantly are plotted against this chondrules ratios. Chondrule 1 was deemed to deviate as $\mathrm{Fe}$ and $\mathrm{Mg}$ seen in the subset sampling of mesostatis in the rim is quite higher than the $\mathrm{Fe}$ and $\mathrm{Mg}$ content seen in chondrule 7. Chondrule 6 has clear differences in chemical patterns and so it was compared to chondrule 7. Chondrule 9 deviated in $\mathrm{Al}$ and $\mathrm{Na}$ content in which the ratios were close to one, while still having a high $\mathrm{Fe}$ and $\mathrm{Mg}$ content.

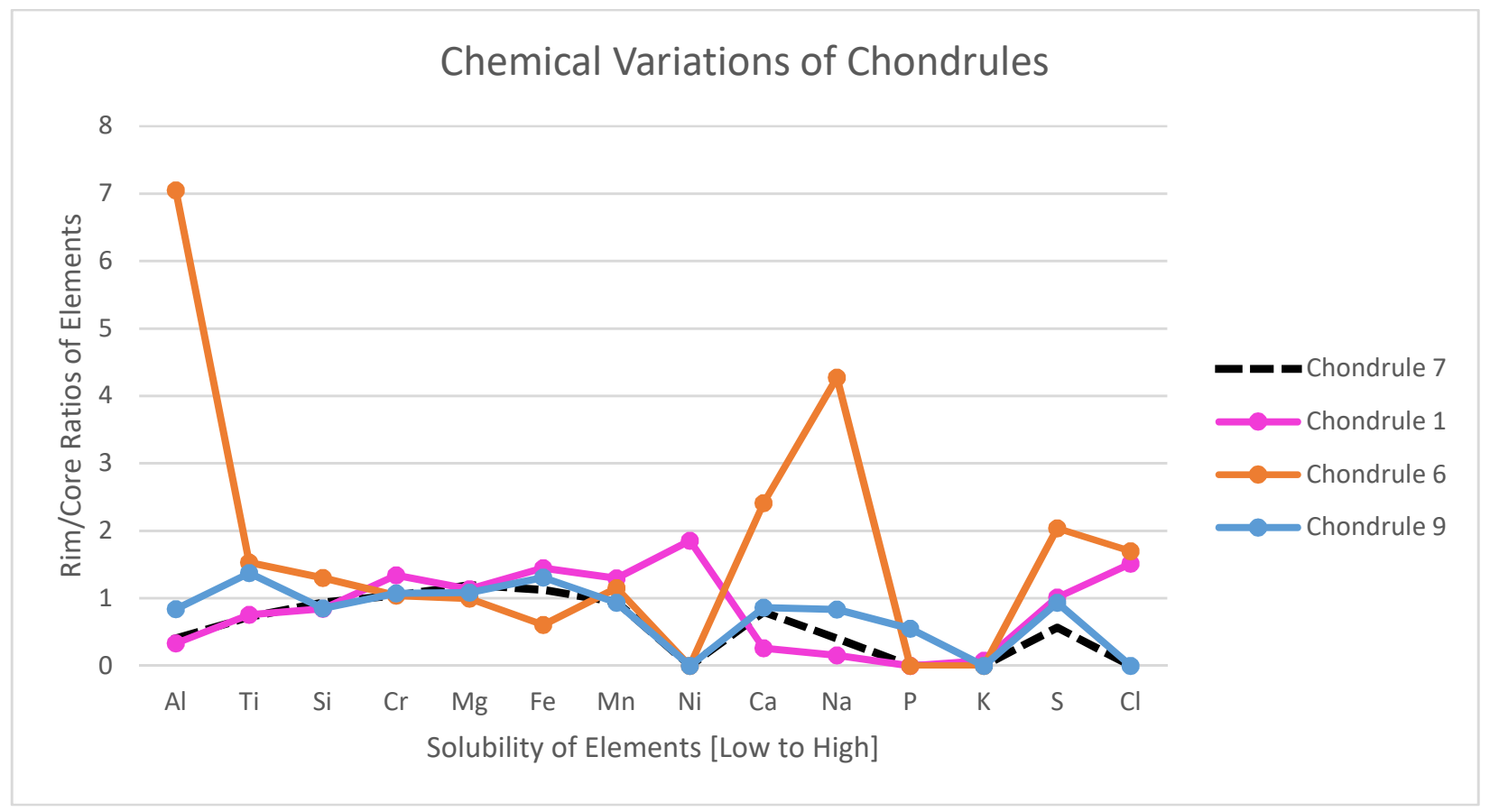

Figure 13: Chondrule chemical data that deviates from the reference chondrule (Chondrule 7) that accurately fits Grossman et al. 's description (dotted black line). 


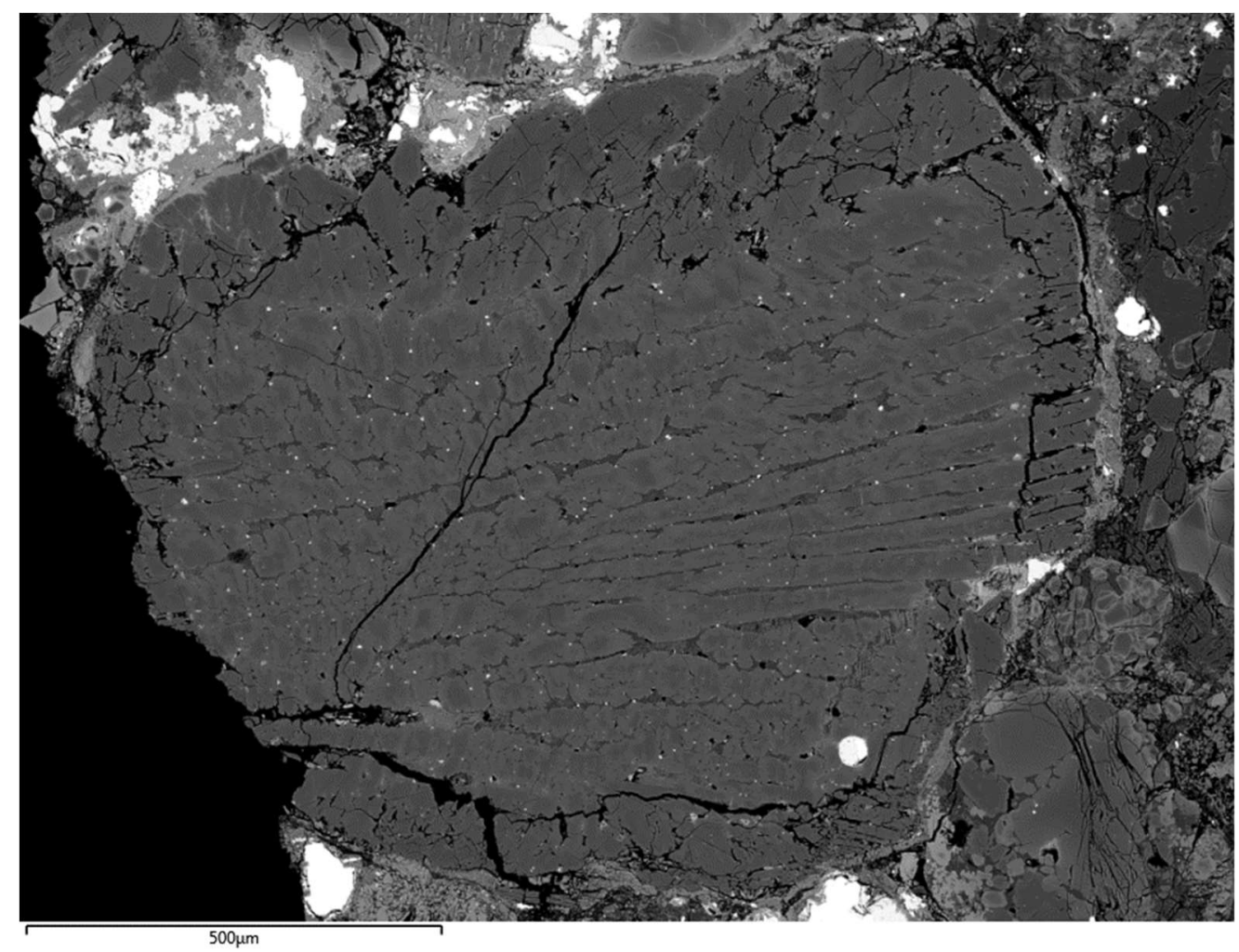

Figure 14: BSE image of architype Chondrule 7 that encapsulates Grossman et al.'s (2000) ideal bleached chondrule. The rim is clearly more porous than the core and the core contains mesostasis throughout.

$\mathrm{Fe} /(\mathrm{Fe}+\mathrm{Mg}) \mathrm{wt} \%$ content was found for each chondrules within both the core and the rim. This was used to compile a ratio between the two values and then compared to the bulk Al ratio of the chondrule [Figure 15]. Similarly, bulk wt\% ratios of $\mathrm{Na}$ were compared against the bulk Al ratios of the chondrules [Figure 16]. This This was done to see how a rather low soluble element, $\mathrm{Fe}$, and rather high soluble element, $\mathrm{Na}$, compared to a quite low soluble element, Al within each chondrule to better understand the possible chemical interaction of aqueous alteration. 


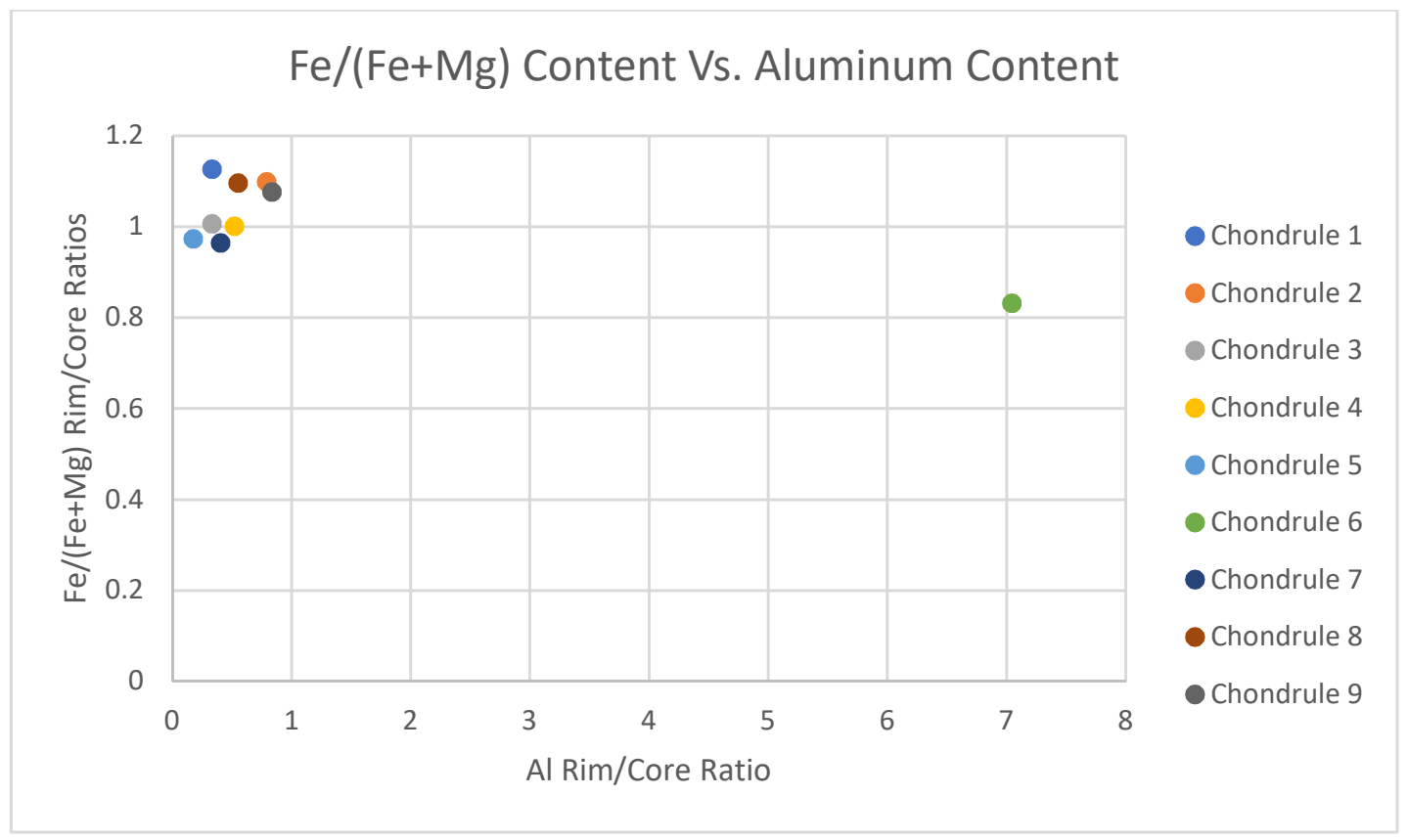

Figure 15: $\mathrm{Fe} /(\mathrm{Fe}+\mathrm{Mg})$ content of each chondrule compared to Al. The majority of chondrules have more $\mathrm{Fe} /(\mathrm{Fe}+\mathrm{Mg}) \mathrm{content}$ within the rim than the core.

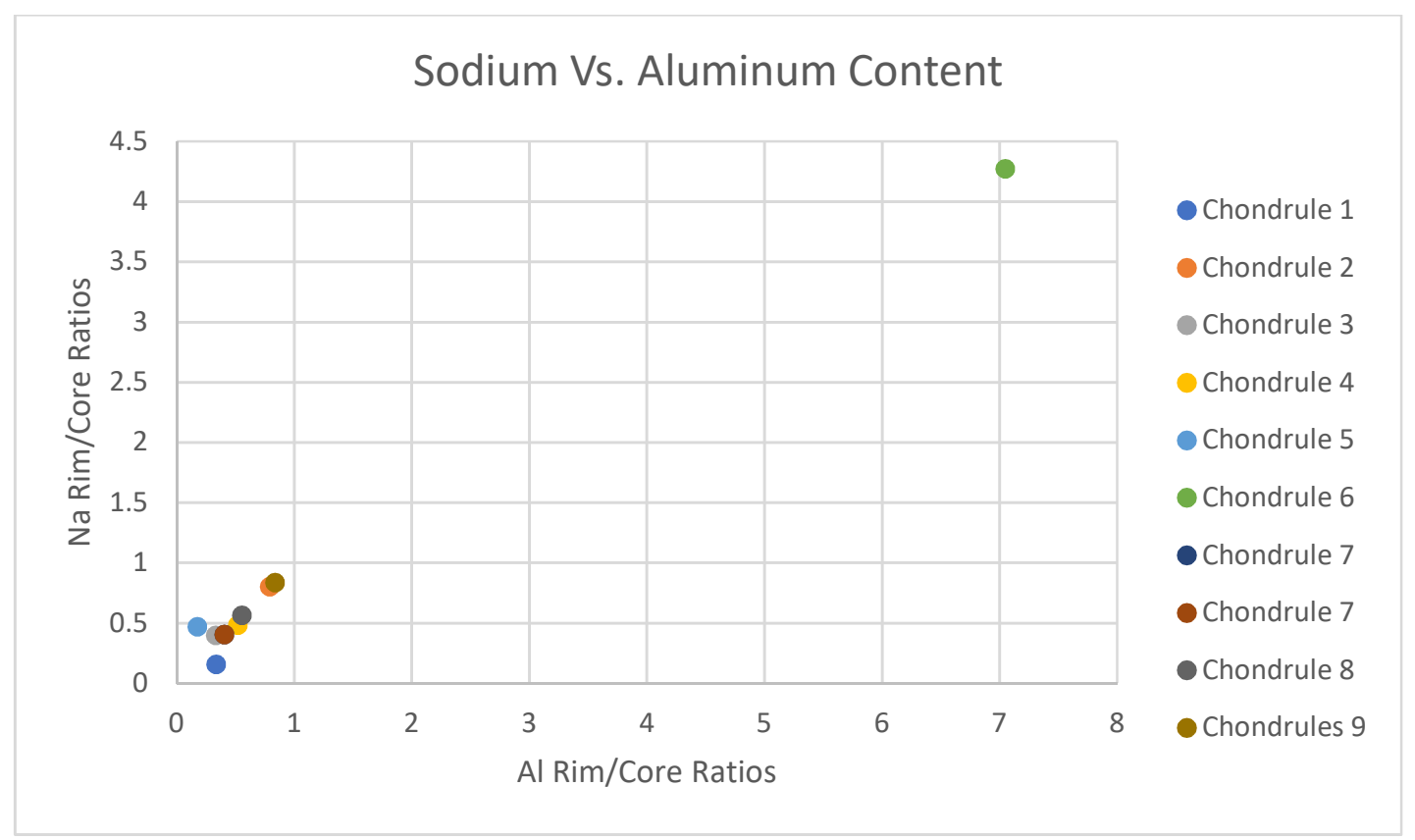

Figure 16: Na ratios of each chondrule compared to Al. All but chondrule 6 have less Na and Al in the rim than the core.

Figure 17 shows how the $\mathrm{Fe} /(\mathrm{Fe}+\mathrm{Mg})$ oxide\% ratios calculated for each chondrule compares to Grossman et al.'s (2000) Chondrules. Grossman et al.'s (2000) Fe/(Fe+Mg) oxide\% ratios were calculated for this report and do not appear in the literature [Table 4]. 


\section{$\mathrm{Fe} /(\mathrm{Fe}+\mathrm{Mg})$ Content Comparison to Grossman et al.'s (2000) Results}

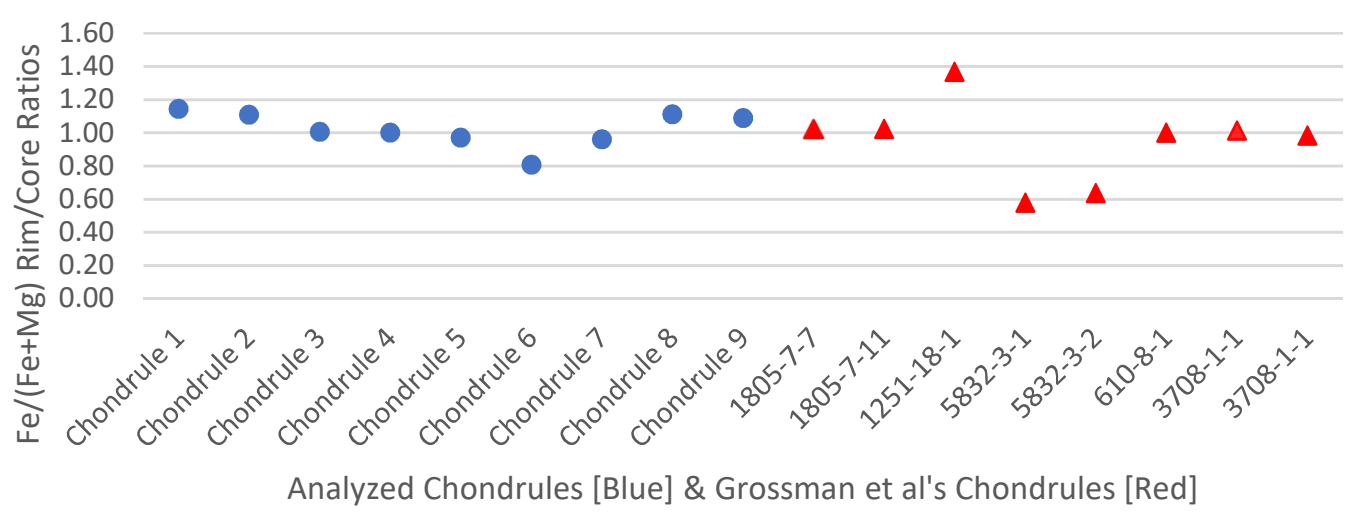

Figure 17: Fe/(Fe+Mg) Oxide \% ratios for the chondrules used in this report [Blue] and the chondrules used in Grossman et al. (2000) [Red]. Note that Grossman et al.'s (2000) chondrule increase in metamorphic grade from left to right.

Table 4: $\mathrm{Fe} /(\mathrm{Fe}+\mathrm{Mg})$ ratios in found in terms of oxide \% to compare to Grossman et al.'s (2000) chondrules.

\begin{tabular}{|l|r|l|r|}
\hline $\begin{array}{l}\text { Chondrules } \\
\text { Anayzed }\end{array}$ & $\begin{array}{l}\text { Fe/(Fe+Mg) } \\
\text { Ratio Value }\end{array}$ & $\begin{array}{l}\text { Grossman et al.'s (2000) } \\
\text { Chondrules }\end{array}$ & $\begin{array}{l}\text { Fe/(Fe+Mg) Ratio } \\
\text { Value }\end{array}$ \\
\hline Chondrule 1 & 1.14 & $1805-7-7$ & 1.02 \\
\hline Chondrule 2 & 1.11 & $1805-7-11$ & 1.02 \\
\hline Chondrule 3 & 1.01 & $1251-18-1$ & 1.37 \\
\hline Chondrule 4 & 1.00 & $5832-3-1$ & 0.58 \\
\hline Chondrule 5 & 0.97 & $5832-3-2$ & 0.64 \\
\hline Chondrule 6 & 0.81 & $610-8-1$ & 1.00 \\
\hline Chondrule 7 & 0.96 & $3708-1-1$ & 1.01 \\
\hline Chondrule 8 & 1.11 & $3708-1-1$ & 0.98 \\
\hline Chondrule 9 & 1.09 & &
\end{tabular}

\section{Discussion:}

The nine bleached chondrules viewed, show quite a large range of results when compared to each other. As mentioned within the results, Chondrule 7 most closely resembles the features described by Grossman et al. (2000) for bleached chondrules. This chondrule has a radial pyroxene texture, has a highly porous rim, contains mesostasis in the core but not in the rim, has a depletion of $\mathrm{Al}$ and $\mathrm{Na}$ in the rim, and just a small increase of $\mathrm{Mg}$ and $\mathrm{Fe}$ in the rim. Chondrule 4 also heavily fits the description used by Grossman et al (2000). This suggests that the samples viewed in this report were indeed bleached. With this being stated, it is rather apparent that not all the chondrules viewed within this report agree with Grossman et al.'s (2000) findings, beyond that of being bleached. The results from each test conducted on the chondrules are shown in Table 5 to show how the chondrules either agree or disagree with Grossman et al. (2000). 
Table 5: Results from each test used to see whether Grossman et al. 's hypothesis is accurate. For the optical test, if it fits with the descriptions given by Grossman et al. (2000) it is said to agree. The porosity test tells which region is more porous. The chemical data section indicates if it agrees with Grossman et al.'s data. The chemistry test $-\mathrm{Fe} /(\mathrm{Fe}+\mathrm{Mg})$ is said to agree if the ratio value in Table 4 is \pm 0.05 from 1. The chemistry test $-\mathrm{Na}$ is said to agree if the ratio is below 1 . The chemistry test-Al a is said to agree if the ratio is below 1.

\begin{tabular}{|c|c|c|c|c|c|c|}
\hline & $\begin{array}{l}\text { Optical } \\
\text { Test }\end{array}$ & $\begin{array}{l}\text { Porosity } \\
\text { Test }\end{array}$ & $\begin{array}{l}\text { Chemical } \\
\text { Composition } \\
\text { Test }\end{array}$ & $\begin{array}{l}\text { Chemistry } \\
\text { Test - } \\
\mathrm{Fe} /(\mathrm{Fe}+\mathrm{Mg})\end{array}$ & $\begin{array}{l}\text { Chemistry } \\
\text { Test - Na }\end{array}$ & $\begin{array}{l}\text { Chemistry } \\
\text { Test - Al }\end{array}$ \\
\hline $\begin{array}{l}\text { Chondrule } \\
1\end{array}$ & $\begin{array}{l}\text { Mostly } \\
\text { Agrees }\end{array}$ & $\begin{array}{l}\text { Agrees - } \\
\text { Rim } \\
\text { Slightly } \\
\text { More } \\
\text { Porous } \\
\end{array}$ & $\begin{array}{l}\text { Slightly } \\
\text { Disagrees - } \\
\mathrm{Fe}, \mathrm{Mg} \text {, and } \\
\mathrm{Cr} \text { are high } \\
\text { in the rim }\end{array}$ & $\begin{array}{l}\text { Disagrees - } \\
\text { Higher in } \\
\text { rim }\end{array}$ & Agrees & Agrees \\
\hline $\begin{array}{l}\text { Chondrule } \\
2\end{array}$ & Agrees & $\begin{array}{l}\text { Disagrees - } \\
\text { Core More } \\
\text { Porous }\end{array}$ & $\begin{array}{l}\text { Mostly } \\
\text { Agrees }\end{array}$ & $\begin{array}{l}\text { Disagrees - } \\
\text { Higher in } \\
\text { rim }\end{array}$ & Agrees & Agrees \\
\hline $\begin{array}{l}\text { Chondrule } \\
3\end{array}$ & Agrees & $\begin{array}{l}\text { Disagrees - } \\
\text { Core More } \\
\text { Porous }\end{array}$ & Agrees & Agrees & Agrees & Agrees \\
\hline $\begin{array}{l}\text { Chondrule } \\
4\end{array}$ & Agrees & $\begin{array}{l}\text { Agrees - } \\
\text { Rim } \\
\text { Slightly } \\
\text { More } \\
\text { Porous }\end{array}$ & $\begin{array}{l}\text { Mostly } \\
\text { Agrees }\end{array}$ & Agrees & Agrees & \\
\hline $\begin{array}{l}\text { Chondrule } \\
5\end{array}$ & Agrees & $\begin{array}{l}\text { Disagrees - } \\
\text { Core More } \\
\text { Porous }\end{array}$ & $\begin{array}{l}\text { Slightly } \\
\text { Disagrees - } \\
\mathrm{K} \text {, and Ca } \\
\text { less } \\
\text { prevalent in } \\
\text { core }\end{array}$ & Agrees & Agrees & Agrees \\
\hline $\begin{array}{l}\text { Chondrule } \\
6\end{array}$ & $\begin{array}{l}\text { Mostly } \\
\text { Agrees }\end{array}$ & $\begin{array}{l}\text { Disagrees - } \\
\text { Core More } \\
\text { Porous }\end{array}$ & $\begin{array}{l}\text { Disagrees - } \\
\mathrm{Al} \text { and } \mathrm{Na} \\
\text { are very high } \\
\text { in the rim }\end{array}$ & $\begin{array}{l}\text { Disagrees- } \\
\text { Lower in } \\
\text { rim }\end{array}$ & Disagrees & Disagrees \\
\hline $\begin{array}{l}\text { Chondrule } \\
7\end{array}$ & Agrees & $\begin{array}{l}\text { Agrees - } \\
\text { Rim More } \\
\text { Porous }\end{array}$ & Agrees & Agrees & Agrees & Agrees \\
\hline $\begin{array}{l}\text { Chondrule } \\
8\end{array}$ & Agrees & $\begin{array}{l}\text { Agrees - } \\
\text { Rim More } \\
\text { Porous } \\
\end{array}$ & $\begin{array}{l}\text { Mostly } \\
\text { Agrees }\end{array}$ & $\begin{array}{l}\text { Disagrees - } \\
\text { Higher in } \\
\text { rim }\end{array}$ & Agrees & Agrees \\
\hline $\begin{array}{l}\text { Chondrule } \\
9\end{array}$ & $\begin{array}{l}\text { Mostly } \\
\text { Agrees }\end{array}$ & $\begin{array}{l}\text { Agrees - } \\
\text { Rim More } \\
\text { Porous }\end{array}$ & $\begin{array}{l}\text { Disagrees - } \\
\text { Fe and Ti } \\
\text { high in the } \\
\text { core. Al and } \\
\mathrm{Na} \text { less } \\
\text { prevalent in } \\
\text { core }\end{array}$ & $\begin{array}{l}\text { Disagrees - } \\
\text { Higher in } \\
\text { rim }\end{array}$ & Agrees & Agrees \\
\hline
\end{tabular}


Shown from Table 5, looking only at the optical, porosity, and chemical tests, it appears that Chondrule 4, 7, and 8 conditionally agree with Grossman et al.'s findings. Chondrule 5 approximately agrees except for a slight increase in K compared to the ideal bleached chondrule and the core have greater porosity than rim. Due to the subset data showing insignificant values of $\mathrm{K}$, this bulk ratio for $\mathrm{K}$ can be ignored meaning only the porosity disagrees with Grossman et al. Chondrule 2 and 3 also have clearly defined porous cores but in all other regards agrees with the ideal bleached chondrule. If the high porosity in the cores for these three chondrules could be disregarded, it could be easily determined that at least six out of the nine chondrules $(2,3,4,5,7$, 8) agree with Grossman et al.'s findings. However, it is quite challenging to explain why the cores of the chondrules are more porous, as these should be less susceptible to alteration and dissolution than the rims, if alteration proceeds from the outside in. This is especially true for Chondrule 2 [Figure 15], which has a well-defined rim with limited cracks making it challenge to address how the water would potentially remove mesostasis from the core instead of the rim and yet still have the chemical composition that mimics Grossman et al.'s bleached chondrule. This along with the fact that Chondrules 2, 3, and 5 do not agree chemically with each other, makes it probable that a slight variation in formation and alteration occurred between the chondrules. It is also likely that these chondrules vary in alteration processes compared to Chondrule 4, 7, and 8 . Chondrules 1, 7, and especially 6 show a rather stark disagreement with Grossman et al. (2000) [Figure 11]. None of these chondrules share a great relationship chemically and seem to have rather unique features that separate them apart. Chondrule 6 is so different from a bleached chondrule as described by Grossman et al. (2000) that it could be considered not to be a bleached chondrule. Optically though, it is a fine candidate for being bleached as it resembles Chondrule 4 and 9.

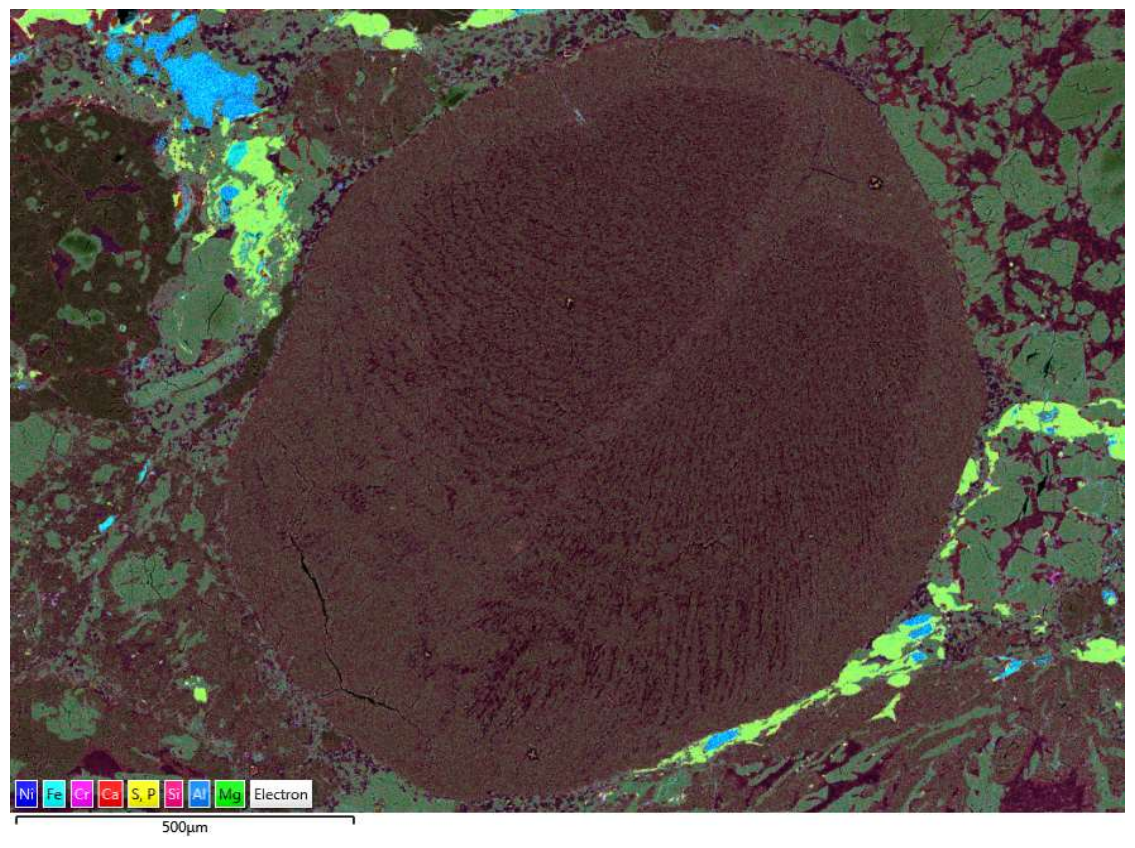

Figure 18: Chondrule 2's false color map showing the defined rim and low porosity present within it.

When viewing the chemical tests of Chondrule 1 it seen that has a $\mathrm{Fe} /(\mathrm{Fe}+\mathrm{Mg})$ content is more prevalent in the rim. Chondrule 8 also shows a high content within the rim meaning that both 
chondrites have bleached chondrules with rather high $\mathrm{Fe} /(\mathrm{Fe}+\mathrm{Mg})$ content which only seen in one of Grossman et al.'s (2000) chondrules. This is important to note as it is stated that low metamorphosed chondrules should have similar quantities of Fe in the rim and core (Grossman et al. 2000). In terms of aqueous alteration and solubility, Fe is rather low in terms of solubility, and so it would seem that more Fe would be present after the influx of water removing possible mesostasis. There is an issue with this though as $\mathrm{Fe} /(\mathrm{Fe}+\mathrm{Mg})$ content is not always more prevalent in the rim for both these chondrules and Grossman et al.'s (2000). This becomes even more challenging to address when it seen that all but one $\mathrm{Al}$ and $\mathrm{Na}$ test agree. In terms of solubility, these two elements should interact with water quite differently. It is seen that the interact almost proportionally to each other in Figure 16 which means shows that indeed solubility cannot play a key role in the depletion of these elements in terms of terrestrial weather. Even more surprising is Chondrule 6 as it has inverse $\mathrm{Al}$ and $\mathrm{Na}$ results compared to the ideal bleached chondrule but hold a similar proportionality. This is another system in which these two elements of contrasting solubility do not agree in terms of aqueous alteration as the sole mechanism of bleaching. It can be seen that most chondrules do agree with the preferential distribution of $\mathrm{Al}$ and $\mathrm{Na}$ being more abundant in the core, but they can also be seen to distribute inversely meaning aqueous chemical alteration alone does not explain this alteration being seen within the chondrules. This means that some physical component of dissolution must have been present during alteration to be able to keep this proportionality consistent.

\section{Conclusion:}

By analyzing nine chondrules across two chondritic meteorites it was found that the common features stated by Grossman et al. (2000) of a bleached chondrule do not always hold true. It was found that although the chondrules viewed agreed optically with Grossman et al.'s research, the porosity and chemical distributions did not. One chondrule, Chondrule 6 (0781-A), was seen to have almost inverse chemical qualities to the optimal bleached chondrule but did agree optically. Of the remaining eight, two chondrules (4 and 8) agreed with Grossman et al.'s findings. Chondrules 3 and 5 only had one main difference in which the porosity was greater in the core. Chondrule 2 had a more porous rim and a higher $\mathrm{Fe} /(\mathrm{Fe}+\mathrm{Mg})$ content than an ideal low metamorphosed bleached chondrule. Chondrule 8 only had a higher $\mathrm{Fe} /(\mathrm{Fe}+\mathrm{Mg})$ content. Chondrules 1 and 9 did not agree with the ideal bleached chondrule although exhibited correct optical representation. Due to the results of this testing specifically the relationship between Al and $\mathrm{Na}$ content it seems unlikely that aqueous alteration is the sole reason behind bleaching seen in chondrules. Since Al was determined to have low solubility and Na was determined to have high solubility, the rims should have exhibited only a loss in $\mathrm{Na}$ and not $\mathrm{Al}$ in terms of aqueous alteration. This is not the case and both elements are depleted in the rim meaning some other method of dissolution had to occur. Future research should be done for additional chondrules the same methodology in this report obtaining ion microprobe data on the chondrules as was done by Grossman et al. (2000) and estimating the conditions necessary to account for the observed fayalite and ferrosilite contents. Additional testing on the matrix of these chondrules could indicate that the dissolution process that occurred, by seeing if high feldspathic minerals surround these chondrules as well. Finally, it is possible that physical dissolution likely occurred 
during the formation of these chondrules and that more than one type of bleaching process may have occurred. 


\section{Work Cited}

Brearley, A., 2006, The Action of Water: Meteorites and the Early Solar System II, University of Arizona Press, Tucson, p. 584-624.

Brigham, C., Yabuki, H., Ouyang, Z., Murrell, M., El Goresy, A., and Burnett, D., 1986, Silicabearing chondrules and clasts in ordinary chondrites: Geochimica et Cosmochimica Acta, v. 50, p. 1655-1666, doi: 10.1016/0016-7037(86)90128-6.

Buggle B., Glaser B., Hambach U., Gerasimenko N., and Slobodan M. (2011) An evaluation of geochemical weathering indices in loess-paleosol studies. Quaternary International, v. $240,12-21$.

Burbine, T., McCoy, T., Meibom, A., Gladman, B., and Keil, K., 2002, Meteoritic Parent Bodies: Their Number and Identification: Asteroids III, University of Arizona Press, Tucson, p.653-667.

Friedrich, J., Perrotta, G., and Kimura, M., 2014, Compositions, geochemistry, and shock histories of recrystallized LL chondrites: Geochimica et Cosmochimica Acta, v. 139, p. 83-97, dio: 10.1016/j.gca.2014.04.044

Goudy, S., and Koolhoven, C., 2018, Bleached Chondrules in NWA 11991: Research paper for G438/538 Scanning Electron Microscopy in the Sciences, fall term 2018 at Portland State University, instructor Dr. Richard Hugo.

Grossman, J., 1998, Radial Pyroxene And Cryptocrystalline Chondrules as Indicators of Aqueous Alteration and Thermal Metamorphism in Ordinary Chondrites: Lunar and Planetary Science XXIX,.

Grossman, J., Alexander, C., Wang, J., and Brearley, A., 2000, Bleached chondrules: Evidence for widespread aqueous processes on the parent asteroids of ordinary chondrites: Meteoritics \& Planetary Science, v. 35, no. 3, p. 467-486, doi: 10.1111/j.19455100.2000.tb01429

Grossman, J., and Brearley, A., 2005, The onset of metamorphism in ordinary and carbonaceous chondrites: Meteoritics \& Planetary Science, v. 40, no. 1, p. 87-122, doi: 10.1111/j.1945-5100.2005.tb00366.

Hubbard, A., and Ebel, D., 2015, Semarkona: Lessons for chondrule and chondrite formation: Icarus, v. 245, p. 32-37, doi: 10.1016/j.icarus.2014.09.025.

Jilly-Rehak, C., Huss, G., Nagashima, K., and Schrader, D., 2017, Low-temperature aqueous alteration on the CR chondrite parent body: Implications from in situ oxygen-isotope analyses: Geochimica et Cosmochimica Acta, v. 222, p. 230-252, dio:

10.1016/j.gca.2017.10.007

Klahr, H., and Schreiber, A., 2015, Linking the Origin of Asteroids to Planetesimal Formation in the Solar Nebula: Proceedings of the International Astronomical Union, v. 10, no. S318, p. 1-8, doi: 10.1017/s1743921315010406. 
Lauretta, D. S., Nagahara H., Alexander, C. M. O'd., 2006, Petrology and Origin of Ferromagnesian Silicate Chondrules, Meteorites and the Early Solar System II, D. S. Lauretta and H. Y. McSween Jr. (eds.), University of Arizona Press, Tucson, 943 pp., p.431-459

Li, Y-H., 2000, A Compendium of Geochemistry—From solar nebula to the human brain, Princeton University Press.

Lindsley, D., Davis, B., and Macgregor, I., 1964, Ferrosilite (FeSiO3): Synthesis at High Pressures and Temperatures: Science, v. 144, p. 73-74, doi: 10.1126/science.144.3614.73.

Millot G., 1970, Geology of Clays-Weathering, Sedimentology, Geochemistry, translated by W.R. Farrand and H. Paquet. Springer-Verlag Wien GmbH. 427 p.

Rubin, A., and Ma, C., 2017, Meteoritic minerals and their origins: Chemie der Erde Geochemistry, v. 77, no. 3, p. 325-385, doi: 10.1016/j.chemer.2017.01.005.

Scott, E., and Krot, A., 2019, Chondrites and Their Components: Treatise on Geochemistry, v. 1, p. $143-200$.

Scott, E., Taylor, G., and Kell, K., 1984, Primitive meteorites: Where do they come from and how do they form?: Press Abstracts from the Fifteenth Lunar and Planetary Science Conference.

Skinner, W., McSween, H., and Patchen, A., 1989, Bleached chondrules and the diagenetic histories of ordinary chondrite parent bodies: Meteoritics, v. 24, p. 326.

Weisberg, M., McCoy, T., and Krot, A., 2006, Systematics and Evaluation of Meteorite Classification: Meteorites and the Early Solar System II: University of Arizona Press, Tucson.

Weisberg, M., and Prinz, M., 1998, Fayalitic olivine in CV3 chondrite matrix and dark inclusions: A nebular origin: Meteoritics \& Planetary Science, v. 33, p. 1087-1099, doi: 10.1111/j.1945-5100.1998.tb01714.

Weyrauch, M., and Bishoff, A., 2012, Macrochondrules in chondrites-Formation by melting of mega-sized dust aggregates and/or by rapid collisions at high temperatures?: Meteoritics \& Planetary Science, v. 47, no. 12, p. 2237-2250, doi: 10.1111/j.1945.2012 .01403

Zolotov, M., Mironenko, M., and Shock, E., 2006, Thermodynamic constraints on fayalite formation on parent bodies of chondrites: Meteoritics \& Planetary Science, v. 41, p. 1775-1796, doi: 10.1111/j.1945-5100.2006.tb00451.x. 